\title{
Reflex regulation of breathing by the paratrigeminal nucleus via multiple bulbar circuits.
}

\author{
Alexandria K Driessen ${ }^{1}$, Michael J Farrell ${ }^{2}$, Mathias Dutschmann ${ }^{3}$, Davor Stanic ${ }^{3}$, Alice E \\ McGovern $^{1}$ and Stuart B Mazzone ${ }^{1 *}$ \\ ${ }^{1}$ Department of Anatomy and Neuroscience, The University of Melbourne, Vic Australia, \\ 3010. \\ ${ }^{2}$ Monash Biomedicine Discovery Institute and Department of Medical Imaging and \\ Radiation Sciences, Monash University, Clayton, Vic Australia, 3800. \\ ${ }^{3}$ The Florey Institute for Neuroscience and Mental Health, The University of Melbourne, Vic
} Australia, 3010.

Corresponding author: Stuart B. Mazzone, $\mathrm{PhD}$, Department of Anatomy and Neuroscience, The University of Melbourne, Vic Australia, 3010. Phone: +61 (03) 8344 6457, e-mail: stuart.mazzone@unimelb.edu.au

\section{Author contribution:}

$\mathrm{AD}$ conducted experiments, contributed to drafting and editing the manuscript. MF contributed to experimental design and manuscript editing. AM conducted experiments, contributed to drafting and editing manuscript. SM conceived experiments, contributed to writing and editing of the manuscript. All authors contributed to the interpretation of the data.

Acknowledgements: This research was supported by grants to Dr SB Mazzone and Dr MJ Farrell from the National Health and Medical Research Council (NHMRC) of Australia [1078943].

\section{Compliance with Ethical Standards:}

Conflict of interest: The authors declare no competing financial interests. 
Animal welfare: Animals were housed in a standard environment and given ad libitum access to water and food. All animal experiments were approved by an accredited institutional Animal Ethics Committee.

\section{Abstract}

Sensory neurons of the jugular vagal ganglia innervate the respiratory tract and project to the poorly studied medullary paratrigeminal nucleus. In the present study we used neuroanatomical tracing, pharmacology and physiology in the guinea pig to investigate the paratrigeminal neural circuits mediating jugular ganglia-evoked respiratory reflexes. Retrogradely traced laryngeal jugular ganglia neurons were largely $(>60 \%)$ unmyelinated and expressed the neuropeptides substance $\mathrm{P}$ and calcitonin gene-related peptide, although a population $(\sim 30 \%)$ of larger diameter myelinated jugular neurons were defined by the expression of vGlut1. Within the brainstem, vagal afferent terminals were confined to the caudal two thirds of the paratrigeminal nucleus. Electrical stimulation of the laryngeal mucosa evoked a vagally-mediated respiratory slowing that was mimicked by laryngeal capsaicin application. These laryngeal reflexes were modestly reduced by neuropeptide receptor antagonist microinjections into the paratrigeminal nucleus, but abolished by ionotropic glutamate receptor antagonists. D,L-homocysteic acid microinjections into the paratrigeminal nucleus mimicked the laryngeal-evoked respiratory slowing, whereas capsaicin microinjections evoked a persistent tachypnoea that was insensitive to glutamatergic inhibition but abolished by neuropeptide receptor antagonists. Extensive projections from paratrigeminal neurons were anterogradely traced throughout the pontomedullary respiratory column. Dual retrograde tracing from pontine and ventrolateral medullary termination sites, as well as immunohistochemical staining for calbindin and neurokinin 1 receptors, supported the existence of different subpopulations of paratrigeminal neurons. Collectively, these data provide anatomical and functional evidence for at least two 
types of post-synaptic paratrigeminal neurons involved in respiratory reflexes, highlighting an unrecognised complexity in sensory processing in this region of the brainstem.

Keywords: Laryngeal reflexes; Jugular vagal ganglia; Airway afferents; Neural crest;

Respiratory sensation 


\section{Introduction}

The respiratory tract is innervated by heterogeneous populations of sensory neurons that respond to a diverse range of chemical and mechanical stimuli, and in turn initiate reflexes and behaviours that optimize breathing and protect the airways. Most of these airway sensory neurons originate from the nodose and jugular vagal ganglia, two embryologically distinct tissues derived from the epibranchial placode and neural crest respectively (Kummer et al., 1992; Nassenstein et al., 2010; D'Autréaux et al., 2011). This embryological distinction appears to drive anatomical, molecular and physiological differences between the airway sensory neurons that comprise the two vagal ganglia (Riccio et al., 1996; Undem et al., 2004; Kwong et al., 2008; Nassenstein et al., 2010; Lieu et al., 2011). Anatomically, for example, the extrapulmonary airways (pharynx, larynx, trachea and mainstem bronchi) are predominately in receipt of afferents from the jugular ganglia, while the intrapulmonary airways and lungs receive substantially more nodose ganglia projections (Undem et al., 2004; McGovern et al., 2015a). In addition, we have shown in both rat (McGovern et al., 2015b) and guinea pig (Driessen et al., 2015) that the central terminal projections of nodose and jugular neurons are different. Thus, nodose ganglia neurons almost exclusively terminate centrally in the medullary nucleus of the solitary tract and integrate with higher brain networks important for limbic and pre-autonomic control, while those from the jugular ganglia project to the medullary paratrigeminal nucleus and are relayed to thalamocortical regions important for somatosensory processing (Driessen et al., 2015; McGovern et al., 2015b). Although the integration of nodose respiratory vagal afferents in the nucleus of the solitary tract has been studied extensively, respiratory vagal sensory processing in the paratrigeminal nucleus has escaped significant attention.

The paratrigeminal nucleus comprises the dorsal component of an interstitial system of neurons embedded in the medullary spinal trigeminal tract (Chan-Paley 1978; Phelan and 
Falls, 1989; Saxon and Hopkins, 1998; Koepp et al., 2006; Panneton et al., 2017), and has been shown to contribute to the processing of nociceptive and baroreceptive inputs (Carstens et al., 1995; Lindsey et al., 1997; Zhou et al., 1999; Yu et al., 2002; Junior et al., 2004; Koepp et al., 2006). Conventional anterograde and retrograde tracing studies in the rat and cat have identified inputs to the paratrigeminal nucleus from the airways, including the larynx, as well as direct projections of paratrigeminal neurons to the nucleus ambiguus, reticular nuclei, nucleus of the solitary tract, facial nucleus, Kölliker-Fuse nucleus and the parabrachial nuclei (Panneton and Burton, 1985; Saxon and Hopkins, 1998; de Sousa Buck et al., 2001; Pinto et al., 2006), which are implicated in respiratory pattern formation and modulation, and associated orofacial behaviours (Dick et al., 1994; Alheid et al., 2004; Dutschmann and Herbert, 2006; Bianchi and Gestreau, 2009; Dutschmann and Dick, 2012; Bautista and Dutschmann, 2014). Stimulation of the larynx evokes a diverse range of nodose and jugular ganglia-dependent airway defence responses that include swallow, apnoea and cough (Canning et al., 2004; Ishikawa et al., 2005; Tsujimura et al., 2013) and we previously reported that laryngeal-induced apnoea in guinea pigs is absent following pharmacological inhibition of the paratrigeminal nucleus (Driessen et al., 2015). In the present study we set out to expand our understanding of vagal afferent processing in the paratrigeminal nucleus by carefully defining the anatomical connectivity of paratrigeminal neurons and characterising in detail the pharmacology of the laryngeal afferent inputs to this nucleus. The findings identify novel complexity to sensory processing within the paratrigeminal nucleus and support the assertion that this nucleus is a functionally important regulator of laryngeal vagal afferent control. 


\section{Methods}

\section{Animals}

Animal experiments, approved by an accredited institutional Animal Ethics Committee, were conducted on adult tri-coloured Dunkin Hartley guinea pigs of either sex ( $\mathrm{n}=82$, weight range $=300-1000 \mathrm{~g})$. Anatomical and physiological data collected from male and female guinea pigs were not different and as such data reflects pooled outcomes from both sexes. Animals were housed in a standard environment and given ad libitum access to water and food.

\section{Neuroanatomical studies}

Conventional and viral tracing of vagal afferents

Guinea pigs were anaesthetised with isoflurane ( $2.5 \%$ in oxygen) via a nose cone and were placed in a supine position on a thermostatically controlled heating pad. Retrograde tracing with fluorescently conjugated cholera toxin subunit B (CT-B ${ }_{594}$, Molecular Probes, C34777) was employed to identify the subpopulations of vagal ganglia neurons that innervate the laryngeal mucosa, following methods described for tracheal afferents (Springall et al., 1987; Riccio et al., 1996; Hunter and Undem, 1999). A small incision was made along the ventral surface of the neck and the muscles overlaying the larynx were retracted. A Hamilton syringe containing $5 \mu \mathrm{l}$ of CT-B $594(2 \mathrm{mg} / \mathrm{ml}$ in $0.9 \%$ saline $)$ was inserted into the laryngeal lumen, distal to the cricotracheal ligament, ensuring that the syringe bevel was facing towards the mucosal surface ( $\mathrm{n}=5$; Figure 1A). Tracer was injected over the course of 30 seconds and after injection the syringe was left in place for five minutes (to prevent backward leakage). Incisions were sutured and animals allowed to recover for 12 days. 
In an additional set of experiments, vagal ganglia neuron termination patterns in the guinea pig brainstem were qualitatively assessed by ganglionic neuron transduction of enhanced Green Fluorescent Protein (eGFP) using an adeno-associated viral vector (AAV8 TRUFr eGFP, UNC Vector Core, Lot \#AV5734B, Viral titre 6.3x10e12). AAV8 serotypes display modest retrograde mobility (Kollarik et al., 2010) and as such can be used to transduce both jugular and nodose neurons by injecting into the nodose ganglia. The animals $(n=4)$ were surgically prepared as outlined above but instead the vagus nerve was identified and blunt dissected from the surrounding tissues, carotid artery and hypoglossal nerve to allow access to the nodose ganglia. Using a micromanipulator, a calibrated glass micropipette (30 $\mu \mathrm{m}$ tip diameter) connected to a pneumatic pressure injector (Pneumatic PicoPump; WPL, Sarasota FL), was positioned parallel to the nerve for clean insertion into the nodose ganglia. $2 \mu 1$ of AAV8 TRUFr-eGFP (AAV-eGFP) was slowly injected unilaterally and swelling of the ganglia was used as an indicator of adequate injection (Figure 1A schematic). Following injection, the micropipette was left in place for an additional five minutes before the incision was sutured and the animal allowed to recover for four weeks.

\section{Anterograde and retrograde tracing of the paratrigeminal nucleus}

Guinea pigs were anaesthetised with isoflurane (2.5\% in medical oxygen) via a nose cone and their heads were placed into a stereotaxic frame at a 45-degree angle. A midline incision was made through the skin, posterior neck muscles and dura mater to expose the medulla at the level between the occipital bone and C1 vertebra. Anterograde tracing with Biotinylated Dextran Amine (BDA) was employed to visualise the terminal projections of paratrigeminal neurons. Using calamus scriptorius as a reference point (approximately $0.5 \mathrm{~mm}$ caudal to Obex and at Bregma $-17.3 \mathrm{~mm}$ ), unilateral microinjections of 250nl of $10 \%$ BDA 
(Sigma Aldrich, B9139) were made into the paratrigeminal nucleus $(n=12)$ using a calibrated glass micropipette $(30 \mu \mathrm{m}$ tip diameter) connected to a pneumatic pressure injector (Pneumatic PicoPump; WPL, Sarasota FL). After injection, the glass micropipette was left in place for five minutes to prevent leakage of the tracer before suturing the incision and allowing the animals to recover for 12 days. It is important to note that microinjections of BDA were made separately into the caudal, central or rostral paratrigeminal nucleus (see Table 1 for stereotaxic coordinates), based on previous analyses in the rat (Pinto et al., 2006). This rostro-caudal distinction was made for operationalisation purposes to best facilitate anatomical and functional studies of the paratrigeminal nucleus.

In a series of separate experiments, we performed dual CT-B retrograde tracing from brainstem regions defined as having both projections from the paratrigeminal nucleus (results from BDA tracing above) and known roles in respiratory control. CT-B 594 and CT-B 488 were unilaterally microinjected (each $250 \mathrm{nl}$ of $2 \mathrm{mg} / \mathrm{ml} \mathrm{CT-B}$ ), one into the Kölliker-Fuse nucleus and the other into the ventral lateral medulla $(n=4)$, or one into the Kölliker-Fuse nucleus and the other into the lateral parabrachial nucleus $(n=3)$. Injections into the ventral lateral medulla were performed as described above, using calamus scriptorius as a reference landmark (refer to Table 1 for stereotaxic co-ordinates). For pontine injections, the head was positioned flat in a stereotaxic frame and holes were drilled in the skull for microinjections at the corresponding coordinates relative to Bregma (Table 1). After each microinjection of CT-B, the micropipette was left in place for five minutes to limit leakage into the surrounding sites. Wounds were sutured and animals allowed to recover for 12 days. 
Tissue harvest, immunohistochemical processing and microscopy

After the required recovery periods, animals were overdosed with sodium pentobarbital (100 mg/kg i.p.) followed by transcardial perfusion with $150 \mathrm{ml}$ of $5 \%$ sucrose in $0.1 \mathrm{M}$ PBS (pH 7.4) and $200 \mathrm{ml}$ of $4 \%$ paraformaldehyde in $0.1 \mathrm{M}$ PBS. Brains, vagal ganglia and the upper airways (specifically the larynx) were dissected. Brains were postfixed overnight in $4 \%$ paraformaldehyde and then cryoprotected in $20 \%$ sucrose at 4 degrees Celsius, while the sensory ganglia and upper airways were stored immediately in $20 \%$ sucrose at 4 degrees Celsius. In the case of tracing from the larynx, prior to further processing, both the larynx and ganglia were cleaned and initially viewed as wholemounts to confirm the presence of the injection site as well as to identify retrogradely traced neurons in the ganglia. In addition, ganglia were also viewed as wholemounts following ganglionic injection of AAV-eGFP to confirm successful injections. All tissues were frozen in OCT embedding compound and cryostat cut sections were either thaw mounted onto gelatin-coated slides across six sets (vagal ganglia, $14 \mu \mathrm{m}$ section thickness), or collected serially in $0.1 \mathrm{M}$ PBS (brain, 50 $\mu \mathrm{m}$ section thickness). An additional four animals were perfused for tissue harvest and immunohistochemical processing of the rostro-caudal extent of the paratrigeminal nucleus to identify the neuronal content in this region.

Brain (free floating) and vagal ganglia (slide-mounted) sections were blocked in $10 \%$ goat or donkey serum in $0.1 \mathrm{M}$ PBS for one hour at room temperature before incubating in the primary antibody of interest (diluted in $2 \%$ goat or donkey serum and $0.3 \%$ Triton-X 100 in 0.1M PBS) for 24-48 hours (see Table 2 for specific antibody details). Tissue sections were then washed three times with 0.1M PBS and incubated in the corresponding secondary antibody for one hour at room temperature. Immunostaining for Forkhead box protein P2 (FoxP2) to identify the Kölliker Nucleus (Stanic et al., 2018), choline acetyl transferase (ChAT) to identify the nucleus ambiguus (McGovern and Mazzone, 2010) and neurokinin 1 
receptor (NK1R) on brain sections was amplified using a tyramide signal amplification kit (Tyramide SuperBoost Kit Alexa Fluor 488-Streptavidin, Thermo Fisher, B40932), following the manufacturer's instructions (see Table 2 for specific antibody details). Tissues containing BDA were further incubated with streptavidin conjugated to Alexa Fluor 594 (strep-594; 1:300) to reveal BDA filled terminal projections. Brain sections were mounted onto gelatincoated slides and coverslipped with an antifade mounting media (Fluroshield, Sigma Aldrich, F6182). All tissues were visualised under appropriate filter cubes using an Olympus BX51 fluorescent microscope and/or a Leica DM6B LED microscope and images were captured using an Olympus DP72 and/or a Leica DFC7000T camera. Representative images were assembled in Adobe Photoshop CS6.

\section{Analysis of neuroanatomical studies}

Immunohistochemical labelling of retrogradely traced laryngeal neurons in the jugular vagal ganglia were quantified by counting immunomarker positive and negative - CT-B 594 positive neurons with clearly identifiable nuclei in each section. Neuronal somal size analyses were conducted by capturing images of CT- $\mathrm{B}_{594}$ traced neurons and measuring their perimeter using a calibrated measuring tool on the Olympus BX51 fluorescent microscope software. These data are presented as frequency distribution histograms for each immunohistochemical marker. The laryngeal traced sensory neurons were characterised by immunostaining for sensory neuron markers including the neuropeptides calcitonin generelated peptide (CGRP) and substance $\mathrm{P}(\mathrm{SP})$, that are characteristic of unmyelinated C-fibre afferents, as well as by the expression of markers of A-fibre afferents (Hermes et al., 2014), which include neurofilament-160kD (NF-160kD) or vesicular glutamate transporter 1 (vGlut1). These distinctions were confirmed by quantitatively assessing the co-expression of 
SP or NF-160kD with vGlut1 on a separate set of (untraced) jugular vagal ganglia sections. Counts of neurons expressing NeuN, the calcium binding protein calbindin or neurokinin 1 receptor $(n=5)$ were conducted on representative brainstem slices for three separate levels (caudal, central and rostral) of the paratrigeminal nucleus, and where appropriate statistically compared using an unpaired student's t-test. AAV-eGFP transduction efficiency was determined by quantifying the proportion of eGFP positive neurons in the jugular and nodose vagal ganglia. The central projections of vagal afferents, revealed by AAV-eGFP transduction and subsequent anti-GFP immunohistochemistry are presented as representative photomicrographs only.

BDA and CT-B injection sites in brainstem target nuclei were assessed in serial sections $(100 \mu \mathrm{m}$ intervals) to identify the central injection locus and the rostro-caudal spread of injectate. Three of the twelve experiments were excluded due to evidence of injectate outside of the paratrigeminal nucleus. BDA-labelled paratrigeminal nucleus projections were subsequently analysed throughout the brainstem using a three-point weighted density scale, similar to that previously employed to analyse anterograde tracing in the rat (McGovern et al., 2015b). Regions of interest across multiple serial tissue sections per animal were assessed at 20x magnification to assign a score that approximates the number of traced terminals within the region $0=0$ fibres, $1=1-50$ fibres (sparse labelling), $2=50-300$ (intermediate labelling), $3=>300$ fibres (dense labelling). An overall peak density score was determined for each region for each animal to allow for semi-quantitative comparisons of paratrigeminal projections across brain regions and to identify those with the most robust labelling. Data are presented graphically in a density connectome diagram in which the weight of the interconnecting line between nuclei reflects the semi-quantitative density of traced terminals, as defined above. In dual retrograde tracing studies, single and double labelled neurons in the paratrigeminal nucleus were quantified by counting CT-B ${ }_{594}$ and CT-B 488 positive cells with a 
clearly identifiable nucleus in each section of the paratrigeminal nucleus, across its rostrocaudal extent. Traced neuron counts were summed across tissue sections and animals to allow a percentage of dual traced neurons relative to the total number of traced neurons to be calculated for each pair of injected regions.

\section{Physiological studies}

In the guinea pig, the laryngeal mucosa is innervated by jugular afferents travelling mostly within the superior laryngeal nerves and nodose ganglia afferents travelling mostly within the recurrent laryngeal nerves (Canning et al., 2004). Thus, surgical transection of the recurrent laryngeal nerves conveniently generates a laryngeal airway preparation that is almost exclusively innervated by jugular afferent fibres (Driessen et al., 2015). All physiological experiments described below were conducted in animals following bilateral resection of the recurrent laryngeal nerves.

Laryngeal stimulation-evoked respiratory responses in anesthetised guinea pigs

Guinea pigs were anesthetised with urethane (1.5g/kg i.p.), the level of which was determined to be adequate by assessing the palpebral and toe withdrawal reflexes. Animals were placed supine on a thermostatically controlled heating pad. A midline incision was made along the ventral surface of the neck and the muscles were retracted to expose the larynx, trachea and underlying nerves and blood vessels. Recurrent laryngeal nerve transection was performed by first blunt dissection of the nerves away from the trachea at the level of the third tracheal ring and then cutting and removing a $1 \mathrm{~cm}$ segment of each nerve to ensure complete denervation. The left carotid artery was cannulated using polyethylene tubing (internal diameter $=0.5 \mathrm{~mm}$ and outer diameter $=0.9 \mathrm{~mm}$ ) attached to a pressure 
transducer filled with heparinised saline (50 U/ml, Sigma Aldrich) to measure arterial blood pressure (ABP) and heart rate (HR). The distal extrathoracic trachea was cannulated and connected via a side-port to a pressure transducer to measure the tracheal pressure (TP) and changes associated with spontaneous respiration. Output from pressure transducers were filtered and amplified (Neurolog Systems, Digitimer, Hertfordshire, UK), digitised (Micro1401 A-D converter, CED, Cambridge, UK) and recorded using Spike II software (CED, Cambridge, UK) for offline analysis. A midline incision was made through the larynx to expose the mucosal surface allowing placement of a platinum bipolar stimulating electrode (Driessen et al., 2015). Less than 7\% of animals (2 out of 30) coughed in response to mechanical stimulation of the larynx after recurrent laryngeal nerve transection, consistent with successful denervation of nodose-derived mechanoreceptors (Canning et al., 2004).

After a 20 minute stabilisation period, electrical stimulation (model s48, Grass Instruments) of the larynx was performed. A voltage response curve was conducted by delivering increasing voltages $(0.1-16 \mathrm{~V})$ to the larynx, at a constant maximum stimulating frequency $(32 \mathrm{~Hz})$, pulse duration $(1 \mathrm{msec})$ and train duration $(10 \mathrm{sec})$. On average the voltage required to elicit $50 \%$ of the maximum response $\left(\mathrm{EV}_{50}\right)$ was $5.1 \pm 0.4 \mathrm{~V}$ and the optimum voltage (i.e. the voltage that evoked a reproducible maximum respiratory response) was $10.9 \pm 0.4 \mathrm{~V}$. In each preparation, the optimum voltage was then used to conduct a frequency response analysis by sequentially increasing the stimulation frequency $(1-32 \mathrm{~Hz})$. Following baseline electrical stimulation of the larynx, guinea pigs were placed onto a stereotaxic frame and the brainstem exposed to allow for bilateral microinjection of the following pharmacological agents into the paratrigeminal nucleus as previously described (Driessen et al., 2015); vehicle ( $0.9 \%$ saline, $\mathrm{n}=7), 5 \mathrm{mM}$ (of each in combination) of CNQX/AP-5 (ionotropic glutamate receptor agonists; $\mathrm{n}=8$ ), $5 \mathrm{mM}$ (of each in combination) of CP99994/ SB222200 (neurokinin 1 and 3 receptor antagonists; n=10) and 2mM BIBN4096 (CGRP 
receptor antagonist; $n=5)$. The appropriateness of the antagonist concentrations employed were determined from previous studies (Bongianni et al., 2005; Mutolo et al., 2008; Hildreth and Goodchild, 2010; Hether et al., 2013). The volume of injection was uniformly 250nl per injection site and each animal received three microinjections bilaterally into the paratrigeminal nucleus (to cover the rostro-caudal extent; see Table 1). Animals were immediately returned to a supine position and the frequency response of electrical stimulation of the larynx reassessed in a paired experimental design. In all instances, the second series of laryngeal stimuli was completed within a period of 30 minutes from the first microinjection. In animals where the respiratory response to laryngeal stimulation appeared unaltered by treatment, the superior laryngeal nerves were subsequently bilaterally transected and the optimum stimulation voltage and frequency reassessed to confirm that all responses were dependent upon laryngeal innervation.

In a separate series of experiments, animals received chemical, rather than electrical, stimulation of the larynx $(n=10)$ by superfusing the laryngeal mucosa with capsaicin (activator of transient receptor potential vanilloid 1 (TRPV1) expressing neurons). A hooked 25-gauge needle was inserted into the intact rostral laryngeal lumen and connected to a $1 \mathrm{ml}$ syringe via polyethylene tubing (internal diameter $=0.5 \mathrm{~mm}$ and outer diameter $=0.9 \mathrm{~mm}$ ). A second 18-gauge cannula was positioned within a small incision at the caudal end of the laryngeal lumen, immediately below the cricotracheal ligament, and connected to gentle vacuum to allow for collection of superfused solutions. Stimuli consisted of $0.5 \mathrm{ml}$ of vehicle or capsaicin $(10 \mu \mathrm{M})$ warmed to 37 degrees Celsius and slowly injected over a 40 second period. 10 minute rest periods between each perfusion ensured any evoked respiratory responses had returned to baseline. The same pharmacological modifications were made in the paratrigeminal nucleus, as described above for the electrical stimulation of the larynx. 
Due to marked tachyphylaxis of capsaicin-evoked responses in this protocol, the study design was necessarily unpaired.

Paratrigeminal stimulation-evoked respiratory responses in anesthetised guinea pigs

To further assess the role of glutamate in respiratory responses mediated via the paratrigeminal nucleus, we performed microinjection of DL-homocysteic acid (DLH, a glutamate analogue) into the caudal, central or rostral paratrigeminal nucleus. We additionally reasoned that microinjections of capsaicin into the paratrigeminal nucleus should activate the central terminals of nearby afferents (Mazzone and Geraghty, 1999; Geraghty and Mazzone, 2002) and thereby evoke responses sensitive to antagonism of glutamate and/or neurokinin receptors. Urethane anesthetised guinea pigs were prepared for physiological monitoring of respiration, blood pressure and heart rate as described above. DLH (50mM; $\mathrm{n}=26$ injection sites) or capsaicin (400nM; $\mathrm{n}=16$ injection sites) were microinjected unilaterally across the rostral-caudal extent of the paratrigeminal nucleus (see stereotaxic coordinates in Table 1) with a five minute period between each to allow for evoked physiological responses to return to baseline. In separate animals, capsaicin responses were assessed in the central paratrigeminal nucleus (the most responsive site) following prior injection of the paratrigeminal nucleus with either CNQX/AP-5 (5mM each in combination; $\mathrm{n}=4)$ or CP99994/SB222200 (5mM each in combination; $\mathrm{n}=5)$.

\section{Analysis of physiological studies}

Respiratory rate, blood pressure and heart rate were calculated from the chart recordings at baseline and following each stimulation frequency or dose of capsaicin. For electrical stimulation, respiratory rates were calculated over 10 second periods immediately 
before (baseline) and during the stimulus train (evoked response) and multiplied by six to determine the equivalent respiratory rate per minute values. For chemical stimulation experiments and capsaicin microinjection into the paratrigeminal nucleus, respiratory rates were similarly calculated over a 10 second period directly before each challenge/injection and again at the time of the peak effect. Alternately, responses evoked by DLH microinjection across the rostro-caudal extent of the paratrigeminal nucleus were assessed over five second periods because this more accurately characterised the highly transient responses observed for this stimulus. Changes in mean arterial blood pressure and heart rate were similarly calculated in all cases before and after the stimulation periods.

Physiological data were normalised to baseline values (before any stimulation) for each animal and then plotted as the mean \pm SEM within each cohort. This removed confounding influences of between animal variations in baseline parameters but importantly preserved the specific stimulus-evoked effects. The normality of each data set was assessed using the Maulchy's test of sphericity and/or Brown-Forsythe test of variances. Each cohort was subsequently assessed for interaction effects of frequency and group using Pillai's trace multivariate test. Post-hoc analyses of the electrical stimulus evoking the maximum reduction in respiratory rate $\left(\mathrm{E}_{\max }\right)$, the electrical frequency required to elicit $50 \%$ of the maximum response $\left(\mathrm{EF}_{50}\right)$ and the peak changes in respiratory rate for each intervention following superfusion of the larynx with capsaicin were conducted using a one-way ANOVA with a least significant differences multiple comparisons test. Respiratory effects evoked by DLH or capsaicin microinjected into the paratrigeminal nucleus were compared to vehicle microinjections using an unpaired student's t-test for each rostro-caudal level of the paratrigeminal nucleus (caudal, central and rostral). Microinjection of the ionotropic glutamate receptors or neurokinin receptors prior to capsaicin injection into the paratrigeminal nucleus were statistically compared using a one-way ANOVA with a least 
significant differences multiple comparisons test. The level for statistical significance was set at $P<0.05$.

\section{Drugs and reagents}

Ionotropic glutamate receptor antagonists CNQX (\#1045 - AMPA receptor antagonist) and AP-5 (\#0105 - NMDA receptor antagonist), Substance P receptor antagonists CP99994 (\#3417 - NK1 receptor antagonist) and SB222200 (\#1393 - NK3 receptor antagonist) and CGRP receptor antagonist BIBN4096 (\#4561) were purchased from Tocris. CNQX and AP-5 were made up in distilled water at stock concentrations of 10mM. CP99994 and SB222200 were made up individually at a stock concentration of $10 \mathrm{mM}$ in a $25 \%$ solution of $100 \%$ ethanol in $0.9 \%$ saline. BIBN4096 was made up at a stock concentration of 50mM in $1 \mathrm{M}$ hydrochloric acid. These stocks were diluted to a $2 \mathrm{mM}$ working concentration with distilled water (and pH adjusted to 7.4). Capsaicin and DLH were purchased from Sigma Aldrich. Capsaicin was made up as a $400 \mathrm{nM}$ or $10 \mathrm{mM}$ working concentration in $10 \%$ ethanol and $10 \%$ tween in $0.9 \%$ saline, for paratrigeminal nucleus microinjection or laryngeal perfusion respectively. For laryngeal perfusion, the $10 \mathrm{mM}$ stock was diluted using $0.9 \%$ saline to create a $10 \mu \mathrm{M}$ working solution, used previously for laryngeal stimulation experiments (Mazzone and Canning, 2002). DLH was made up as $50 \mathrm{mM}$ stock solutions in distilled water (and $\mathrm{pH}$ adjusted to 7.4). In preliminary experiments, all vehicles were tested by microinjection into the paratrigeminal nucleus and shown to have no effect on physiological parameters measured, and as a result saline was used as the standard control for comparison purposes. 


\section{$\underline{\text { Results }}$}

\section{Characterisation of jugular ganglia laryngeal sensory neurons}

CT-B 594 injections into the larynx labelled $224 \pm 73.7$ neurons per jugular ganglia $(n=5$; Figure 1A). Traced neurons displayed a wide range of somal sizes (Figure 1B) consistent with labelling of both $\mathrm{C}$ and A-fibre laryngeal afferent subtypes. Immunohistochemical analysis of the traced jugular ganglia neurons also confirmed this (Figure 1B). Thus, a little over half were small-to-medium sized peptidergic laryngeal traced neurons that immunostained for the neuropeptides CGRP $(58 \%$ positive; average perimeter $=$ $65.7 \pm 2.0 \mu \mathrm{m})$ or $\mathrm{SP}(54 \%$ positive; average perimeter $=67.1 \pm 1.9 \mu \mathrm{m})$, that is representative of the peptidergic C-fibre population (Riccio et al., 1996; Undem et al., 2004). On the other hand, one third of the laryngeal traced neurons were larger in size and immunostained for NF-160kD (21\% positive; average perimeter $=90.5 \pm 3.5 \mu \mathrm{m})$ or vGlut $1(31 \%$ positive; average perimeter $=79.1 \pm 2.8 \mu \mathrm{m}$ ), which are representative of the A-fibre population. Consistent with this, we observed very minimal colocalization of vGlut 1 with the neuropeptide SP ( 7\%), but substantial overlap of vGlut1 with NF-160kd ( 55\%) in untraced vagal ganglia tissues $(\mathrm{n}=4)$.

\section{Anatomical characterisation of the rostro-caudal organisation of the paratrigeminal} nucleus

To our knowledge, the local architecture and rostro-caudal extent of the paratrigeminal nucleus has not been previously described in the guinea pig. NeuN immunohistochemistry revealed an interstitial neuronal group embedded in the dorsal tip of the trigeminal tract that extended from 0.1 to $1.4 \mathrm{~mm}$ rostral to calamus scriptorius (defined herein as the 
paratrigeminal nucleus). The density of neurons was highest in the caudal and central regions of the paratrigeminal nucleus and diminished appreciably in the rostral-most extent. Given that the jugular ganglia contains both $\mathrm{C}$ - and A-fibre laryngeal afferents (Figure 1B), we subsequently assessed the distribution of SP (C-fibres) and vGlut1 (A-fibres) immunolabelling, notably observing apparent topographical distinctions between the location of these two markers in and around the paratrigeminal nucleus (Figure 1C).

AAV-eGFP injections into the nodose ganglia transduced approximately $40 \%$ of nodose neurons and $30 \%$ of jugular neurons, resulting in distributed eGFP labelling of afferent terminals in the brainstem. Not surprisingly these terminations were distributed bilaterally (ipsilateral predominance) in the nucleus of the solitary tract from $0.2 \mathrm{~mm}$ caudal to $1.8 \mathrm{~mm}$ rostral of the calamus scriptorius and importantly, terminal labelling was also evident ipsilaterally in and around the dorsal tip of the spinal trigeminal tract (Ciriello et al., 1981; Altschuler et a., 1989; McGovern et al., 2012). Furthermore, labelling was in close apposition to paratrigeminal neurons (NeuN positive neurons) at the caudal and central levels of the paratrigeminal nucleus but almost absent in the rostral-most extension of the nucleus (Figure 1C). Vagal afferent terminal labelling was absent from the paratrigeminal nucleus in one experiment where AAV injections into the nodose ganglia efficiently transduced nodose neurons but failed to transduce any jugular ganglia neurons, consistent with our previous data showing that vagal projections to the paratrigeminal nucleus originate from jugular neurons exclusively (Driessen et al., 2015). Sparse ipsilateral labelling of terminals was also seen in the ventral geniculate trigeminal nucleus, interpolar part of the spinal trigeminal nucleus and the lateral edge of the caudal part of the spinal trigeminal nucleus, as well as in the medullary reticular formation, including in the region of the nucleus ambiguus, indicative of widely distributed vagal afferent terminations in the brainstem. No contralateral labelling was observed in any brainstem regions outside of the nucleus of the solitary tract. 


\section{Respiratory effects of electrical and chemical stimulation of the larynx}

Prior to any interventions of the paratrigeminal nucleus, baseline respiratory rate averaged $42.4 \pm 1.1$ breaths/minute $(n=30)$. In vehicle treated animals, electrical stimulation of the larynx (at an optimal voltage) produced a frequency dependent slowing of respiration (typically accompanied by visible swallows; not quantified) progressing to complete apnoea at higher stimulation frequencies (Figure 2A). The maximum reduction in respiratory rate $\left(E_{\max }\right)$ following bilateral injection of vehicle into the paratrigeminal nucleus was $40.3 \pm 4.1$ breaths/minute and the effective electrical frequency required to elicit $50 \%$ of the maximum response $\left(\mathrm{EF}_{50}\right)$ was $4.3 \pm 2.1 \mathrm{~Hz}$ (Figure $2 \mathrm{~B}$, Table 3 ). A statistically significant interaction was detected between pharmacological treatment and electrical frequency dependent slowing in respiration $(\mathrm{F}(14,126)=4.02, p<0.0001)$, indicative of one or more of the drug interventions modifying the magnitude of the evoked reflex. Post-hoc evaluations revealed that treatment with the neurokinin receptor antagonists CP99994/ SB222200 significantly reduced the $\mathrm{E}_{\max }(p=0.007)$, but not the $\mathrm{EF}_{50}$, compared to vehicle (Figure 2C, Table 3), although this effect was significantly smaller than that induced by the ionotropic glutamate receptor antagonists $\mathrm{CNQX} / \mathrm{AP}-5$ which inhibited both the $\mathrm{E}_{\max }$ and $\mathrm{EF}_{50}$ compared to vehicle $\left(\mathrm{E}_{\max } p=0.0002 ; \mathrm{EF}_{50} p=0.01\right)$ and the neurokinin receptor antagonists $\left(\mathrm{E}_{\max } p=0.032 ;\right.$ $\mathrm{EF}_{50} p=0.02$, Figure 2C, Table 3). Microinjection of the CGRP receptor antagonist BIBN4096 into the paratrigeminal nucleus alone (Table 3) or in combination with the neurokinin receptor antagonists (data not shown) failed to further inhibit electrically evoked respiratory responses. Heart rate and blood pressure effects evoked by electrical stimulation of the larynx were not significantly altered between pharmacological modifications of the paratrigeminal nucleus (data not shown). 
Chemical stimulation of the larynx using capsaicin superfusion evoked respiratory effects comparable to that seen with electrical stimulation. Following bilateral microinjection of vehicle into the paratrigeminal nucleus, capsaicin superfusion on the larynx consistently produced visible swallowing (data not quantified) and decreased respiratory rate on average by $22 \pm 2.0$ breaths/minute from baseline (often occurring with a brief period of apnoea; Figure 2C). Comparable to the observations with electrical stimulation, capsaicin-induced respiratory slowing was not significantly altered by prior injections of neurokinin receptor antagonists into the paratrigeminal nucleus but was abolished $(p=0.02)$ by glutamate receptor antagonists (Figure 2C).

\section{Respiratory effects of glutamate and capsaicin microinjections into the paratrigeminal nucleus}

Microinjections of the glutamate analogue DLH or capsaicin into the paratrigeminal nucleus evoked opposing effects on respiration. That is, while DLH resulted in an abrupt reduction in respiratory drive (leading to brief arrest of breathing in three cases; Figure 3A, B), capsaicin initiated a slowly developing and persistent increase in respiratory rate (tachypnoea; Figure 3C, D). Although respiratory slowing was induced by DLH across the entire rostro-caudal extent of the paratrigeminal nucleus, the response was only statistically significant for the caudal level of the nucleus compared to vehicle injections (DLH decreased respiratory rate by $9.0 \pm 5.4$ breaths/ minute versus vehicle that increased respiratory rate by $4.5 \pm 3.2$ breaths/ minute $p=0.049$, Figure 3B). On the other hand, capsaicin-induced tachypnoea was significantly different compared to vehicle when injections were made into the central paratrigeminal nucleus (capsaicin increased respiratory rate by $22.5 \pm 5.1$ versus vehicle that increased respiratory rate by $0 \pm 3.8$ breaths/ minute respectively $p=0.009$, Figure 
3D). A comparable response was only observed in two out of the seven cases following microinjection of capsaicin into the caudal paratrigeminal nucleus, whereas capsaicin was without respiratory effect at the level of the rostral paratrigeminal nucleus (Figure 3D). At the level of the central paratrigeminal nucleus, capsaicin-evoked tachypnoea was not significantly altered by prior treatment with the glutamate receptor antagonists (CNQX/AP-5 increased respiratory rate by $18 \pm 4.9$ breaths/ minute, $p=0.39$, Figure $3 \mathrm{E})$, but was abolished following pre-treatment with neurokinin receptor antagonists $(p=0.001$, Figure $3 \mathrm{E})$. These data are consistent with a role for neuropeptides, and not glutamate, in centrally administered capsaicin-evoked tachypnoea. DLH and capsaicin produced small and inconsistent effects on heart rate and blood pressure parameters that were not significantly different between any groups.

\section{Neuroanatomical connectivity of the paratrigeminal nucleus}

Conventional anterograde tracing using microinjections of BDA into the paratrigeminal nucleus was successful in 9 cases, in which the average spherical spread of tracer was $0.8 \pm 0.2 \mathrm{~mm}$ in diameter (Figure 4A). Anterograde labelling of terminals was broadly distributed across many bulbar nuclei, although they were always confined exclusively ipsilateral to the injection site. Suprabulbar projections from the paratrigeminal nucleus, which have been described in rats (Saxon and Hopkins, 1998; Caous et al., 2001; McGovern et al., 2015b), were not observed in the guinea pig (not shown). A similar pattern of BDA labelled terminal projections were observed regardless of the rostro-caudal location of the BDA injection site (Figure 4B). Common termination sites included the medullary spinal trigeminal nucleus, the nucleus of the solitary tract, the ventrolateral medulla in the region of the nucleus ambiguus and pre-Bötzinger complex (Figure 4C), the principal sensory 
trigeminal nucleus and dense projections to the medial and lateral parabrachial nuclei and Kölliker-Fuse nucleus (Figure 4D). However, the strength of connectivity to these common bulbar termination sites was not equal for caudal, central and rostral regions of the paratrigeminal nucleus. Indeed, neurons in the caudal paratrigeminal nucleus displayed relatively strong connectivity with both medullary and pontine targets whereas neurons in the central and rostral paratrigeminal nucleus appear to preferentially project to pontine nuclei (Figure 4B).

\section{Dual retrograde labelling of paratrigeminal neurons from the dorsolateral pons and ventral lateral medulla}

Dual microinjections of CT-B into the Kölliker-Fuse and ventrolateral medulla or Kölliker-Fuse and lateral parabrachial nucleus were successful in 7 out of 16 animals. The central locus of the injection sites and diameter of injectate spread were as follows; KöllikerFuse nucleus $9.4 \pm 0.1 \mathrm{~mm}$ caudal to Bregma and a rostro-caudal spread of $0.3 \pm 0.1 \mathrm{~mm}$, lateral parabrachial nucleus $9.5 \pm 0.2 \mathrm{~mm}$ caudal to Bregma and a $0.3 \pm 0.1 \mathrm{~mm}$ rostro-caudal spread for and the ventrolateral medulla $0.6 \pm 0.1 \mathrm{~mm}$ rostral to calamus scriptorius and a rostro-caudal spread of $1.6 \pm 0.4 \mathrm{~mm}$. Dual CT-B injections into the Kölliker-Fuse and lateral parabrachial nucleus individually labelled a total of 305 and 315 neurons, respectively in the paratrigeminal nucleus (summed across all animals; Figure 5A). Similarly, dual tracing from the Kölliker-Fuse and ventrolateral medulla individually labelled 553 and 535 neurons, respectively in the paratrigeminal nucleus (Figure 5A). In both experimental groups we observed a small proportion of dual labelled neurons within the paratrigeminal nucleus. Only $8 \%$ of neurons ( 81 out of a total of 1007 individually traced neurons) were dual labelled from the Kölliker-Fuse and ventrolateral medulla and $17 \%$ of neurons (91 out of a total of 529 
individually traced neurons) were dual labelled from the Kölliker-Fuse and lateral parabrachial nucleus (Figure 5A).

In separate experiments using immunohistochemistry we identified calbindin positive neurons in the paratrigeminal nucleus, as described previously in the rat (Ma et al., 2005). However, across the rostro-caudal extent of the paratrigeminal nucleus we noted that calbindin positive neurons constituted a significantly smaller population $(p=0.009)$ of paratrigeminal neurons compared to those labelled with the pan neuronal marker NeuN (compare Figure 5B with Figure 1C for NeuN staining). Double immunostaining for calbindin and the neurokinin 1 receptor revealed two distinct populations of neurons in the paratrigeminal nucleus. Indeed, while calbindin and the neurokinin 1 receptors were expressed in a total of 264 and 398 individual neurons in the same tissue sections, only $5.7 \%$ (40 out of 702 counted neurons) were co-labelled with both calbindin and the neurokinin 1 receptor immunoreactivity (Figure 5B). 


\section{Discussion}

We have previously reported the existence of a novel respiratory sensory processing pathway that arises from jugular vagal ganglia afferents and principally innervates the larger airways and projects to the medullary paratrigeminal nucleus (McGovern et al., 2012; Driessen et al., 2015; McGovern et al., 2015a; McGovern et al., 2015b). Ascending projections from paratrigeminal neurons in receipt of airway jugular ganglia inputs terminate in somatosensory processing regions of the brain (McGovern et al., 2015b), which has led us to propose that this sensory circuit may be important for discriminative processing of sensations arising from the large airways. In the present study we investigated the reflexive regulation of respiration mediated by the paratrigeminal nucleus and demonstrate a level of complexity in paratrigeminal sensory integration that has not been previously recognised. Notably, we report functional and anatomical evidence for the existence of at least two distinct postsynaptic targets for afferent inputs in the paratrigeminal nucleus, which likely mediate opposing effects to respiratory drive. Collectively, these data advance our understanding of viscerosensory processing in the brainstem by highlighting a novel vagal afferent neural circuit with features that are consistent with an important role in respiratory sensation, breathing control and pulmonary defense.

\section{A neuroanatomical framework for laryngeal sensory-evoked respiratory responses via the paratrigeminal nucleus}

The laryngeal mucosa is innervated by vagal sensory neurons, the activation of which induces the modulation of respiration and induction of protective expiratory responses that collectively serve to limit the effects of foreign material passing the glottis. However, the neural circuits underpinning afferent processing from this site have not been fully elucidated. 
In the guinea pig, the laryngeal mucosa is in receipt of sensory fibres arising from both the nodose and jugular vagal ganglia (Canning et al., 2004; Undem et al., 2004; McGovern et al., 2015a). Nodose neurons give rise to low threshold laryngeal mechanoreceptors that are important for defensive coughing and expiratory responses evoked by particulate matter and other mechanical stimuli. These neurons project exclusively to the nucleus of the solitary tract and were purposefully excluded from investigations in the present study but have been previously interrogated in detail (Canning et al., 2004; Chou et al., 2008; Mazzone and McGovern, 2008; Canning and Mori, 2011). Conversely, jugular ganglia neurons innervating the laryngeal mucosa are not particularly mechanically sensitive but instead they respond to an array of chemical mediators of which the most notable is capsaicin (Riccio et al., 1996; Canning et al., 2004; Undem et al., 2004; Lieu et al., 2011). Our present data confirms previous studies in guinea pigs identifying a larger population of $\mathrm{C}$-fibre and smaller population of $A \delta$-fibre laryngeal jugular ganglia neurons, differentiated in retrograde tracing experiments by neuron somal size, peptidergic expression markers, myelination and vGlut1 (Riccio et al., 1996; Pedersen et al., 1998; Canning et al., 2004; Mazzone and McGovern, 2008; Hermes et al., 2014).

We have previously shown in both rats and guinea pigs that jugular ganglia neurons project exclusively to the paratrigeminal nucleus and not the nucleus of the solitary tract (Driessen et al., 2015; McGovern et al., 2015b). Perhaps best studied in the rat, the paratrigeminal nucleus is part of an extensive interstitial system of the trigeminal nucleus (Chan-Paley 1978, Phelan and Falls, 1989; Panneton et al., 2017) and receives additional inputs from trigeminal and spinal nociceptive afferents (Ma et al., 2007; Alioto et al., 2008). Neuronal tracing studies in rats have shown output projections from paratrigeminal neurons to bulbar nuclei involved in autonomic and nociceptive processing, including the ventral lateral medulla and pontine nuclei (Feil and Herbert, 1995; de Sousa Buck et al., 2001; Pinto 
et al., 2006; McGovern et al., 2015b). Our data in the guinea pig is largely consistent with the rat in that we noted caudal to rostral differences in the organisation of the paratrigeminal nucleus and its vagal inputs, as well as comparable paratrigeminal pontomedullary connectivity. However, unlike the rat (McGovern et al., 2015b), guinea pig paratrigeminal neurons did not project to contralateral medullary nuclei or directly to the ventrobasal thalamus. Additionally, although the rat paratrigeminal nucleus projects heavily to the caudal sub-nucleus of the nucleus of the solitary tract (de Sousa Buck et al., 2001; McGovern et al., 2015b), this was not evident in the guinea pig and instead we observed that such connectivity was confined to rostral subnuclei. This argues that the paratrigeminal nucleus in the guinea pig likely acts independently of the nucleus of the solitary tract in the initial processing of cardiorespiratory-related primary afferent inputs to the brainstem.

\section{Functional and anatomical evidence for multiple paratrigeminal processes regulating respiration}

Our present data confirms our previous findings showing that laryngeal afferents arising from the jugular ganglia evoke respiratory slowing when activated, which is a reflex mediated by afferent inputs to the paratrigeminal nucleus (Driessen et al., 2015). We have extended our previous findings by demonstrating a primary role for glutamatergic neurotransmission in the paratrigeminal nucleus in laryngeal jugular afferent evoked respiratory responses, regardless of whether reflexes were evoked by electrical or chemical stimulation of the laryngeal mucosa. However, we also consistently noted a relatively smaller but significant effect of blocking neurokinin receptors, but not CGRP receptors, on this reflex evoked respiratory slowing. The principle role of glutamatergic neurotransmission is also supported by the stimulus parameters and kinetics of the evoked responses, especially during 
electrical stimulation. Thus, large respiratory effects were evoked at low stimulation intensities, and proceeded with a rapid (almost immediate) onset and terminated equally rapidly after cessation of stimulation, consistent with ionotropic rather than metabotropic neurotransmission. However, although our studies are consistent with glutamate being the primary excitatory transmitter of laryngeal afferents in the paratrigeminal nucleus, with neurokinins acting as neuromodulators, our data do not allow for the relative contribution of A $\delta$-fibre versus C-fibre laryngeal afferents to be determined. Indeed, almost all vagal ganglia neurons express vGlut2, suggesting that all are likely capable of releasing glutamate when activated (Juranek and Lembeck, 1997; Corbett et al., 2005; Mazzone and McGovern, 2008; Fenwick et al., 2014; Rogoz et al., 2015), and as yet a selective stimulus of either A- or Cfibre jugular afferents has not been identified. Nevertheless, the low threshold voltages required to elicit reflex respiratory slowing might suggest a prominent role for jugular Afibres, which to our knowledge, is the first report of a reflex specifically involving this airway afferent subtype.

Several lines of evidence suggest that the processing of afferent inputs within the paratrigeminal nucleus may be more complex than the simple notion that multiple afferent subtypes converge onto common postsynaptic paratrigeminal relay neurons. In this regard, the organisation of afferent inputs to the paratrigeminal nucleus may resemble the nonconvergent specificity of A- and C-fibre vagal afferent synapses that has previously been reported in the nucleus of the solitary tract (Bailey et al., 2002; McDougall and Andresen, 2013). Indeed, whilst microinjection of a glutamate analogue predictably mimicked the effect of laryngeal stimulation by evoking a transient interruption of respiration (presumably secondary to the activation of postsynaptic targets), capsaicin microinjection into the paratrigeminal nucleus paradoxically enhanced respiratory drive. Capsaicin is expected to activate the central presynaptic terminals of afferents in the paratrigeminal nucleus, releasing 
neurotransmitter that subsequently activates paratrigeminal second order neurons (Mazzone and Geraghty, 1999; Fenwick et al., 2014; Hofmann and Andresen, 2016). Although we cannot discount a role for glutamate our data suggest that, under the circumstances employed, neuropeptides are the primary neurotransmitter mediating the centrally administered capsaicin-evoked response given that the tachypnoea was inhibited by neurokinin receptor antagonists but not by ionotropic glutamate receptor antagonists. Of course, centrally injected capsaicin is expected to activate more than laryngeal afferent terminals in the paratrigeminal nucleus. Indeed, the paratrigeminal nucleus is also in receipt of spinal, trigeminal and glossopharyngeal afferents (Cirello et al., 1981; Panneton and Burton, 1981; Panneton and Burton, 1985; Altschuler et al, 1989) and these could conceivably evoke changes in cardiorespiratory behaviours given the extensive projections of the paratrigeminal nucleus to brainstem nuclei important in modulating these effects. However, the opposing respiratory responses evoked by peripheral (laryngeal) versus central afferent activation as well as the rostro-caudal differences noted in functional responsive sites within the paratrigeminal nucleus suggest that several subsets of respiratory modulating paratrigeminal neurons may exist. It remains unclear as to why centrally administered capsaicin evokes respiratory responses that do not rely on glutamatergic neurotransmission in the paratrigeminal nucleus, as it is known that both $\mathrm{A} \delta$ - and C-fibre laryngeal afferents are capsaicin sensitive (Riccio et al., 1996; Canning et al., 2004) and action potential dependent afferent responses are largely glutamatergic. Conceivably, the local calcium events in the afferent terminal differ between the activation of voltage gated (action potential dependent) and TRP (capsaicin dependent) channels such that different pools of small and large dense core neurotransmitter vesicles are mobilized (Fawley et al., 2016; Hofmann and Andresen, 2016). However, such speculation requires further investigation. Regardless, transmitter release under these circumstances is presumably at different synapses given that we observed opposing effects on respiration. 
Our dual retrograde neuronal tracing studies and immunohistochemical staining provide further evidence to support the existence of multiple postsynaptic neuron subtypes within the paratrigeminal nucleus. Very few paratrigeminal neurons were dual labelled following retrograde tracing from the Kölliker-Fuse and ventrolateral medulla, or the Kölliker-Fuse and lateral parabrachial nucleus. This contrasts with some previous finding in rats, which failed to show distinct paratrigeminal neuron populations following dual retrograde tracing from the nucleus of the solitary tract and parabrachial nuclei, ventrobasal thalamus and nucleus of the solitary tract and the ventrobasal thalamus and the parabrachial nuclei (Saxon and Hopkins, 1998). However, we acknowledge that the discrete microinjections employed for this experiment would reduce tracing efficiency and thus our data may over-estimate apparent segregation. Nevertheless, we also demonstrated that at least two different populations of paratrigeminal neurons could be identified based on their immunoreactivity for either calbindin or the neurokinin 1 receptors. Taken together, and considering our functional data, these findings collectively support the hypothesis that types of post-synaptic neurons process afferent inputs in the paratrigeminal nucleus. Whether A- and C-fibre laryngeal afferent inputs are segregated into parallel processing lines onto distinct postsynaptic recipient neurons within the paratrigeminal nucleus, as has been reported for vagal afferent inputs to the nucleus of the solitary tract (Alheid et al., 2011), remains to be resolved.

\section{Physiological significance and conclusion}

In the present study, we employed neuroanatomical tracing as well as physiological and pharmacological experiments to assess the organisation of laryngeal afferent dependent responses mediated by jugular ganglia inputs to the paratrigeminal nucleus. The reflex changes in breathing pattern evoked by laryngeal stimulation or by centrally administered 
agents into paratrigeminal nucleus undoubtedly reflect the intimate connectivity of this nucleus with bulbar regions important for respiratory pattern regulation. Our data reveal an underappreciated connectivity of the paratrigeminal nucleus with pontomedullary nuclei of the respiratory rhythm and pattern generating circuit (Feldman et al., 2003; Dutschmann and Dick, 2012; Ramirez et al., 2012; Smith et al., 2013) and in turn provide a novel perspective to this field. In particular, the pronounced ascending projections into pontine respiratory nuclei that are critical for the modulation of airway resistance, respiratory airflow and the mediation of airway reflexes (Dutschmann and Dick, 2012) underline the significance of this pathway in sensory-motor controls of the upper airways. However, given that jugular ganglia afferents comprise part of the neural crest-derived cranial ganglia (Kwong et al., 2008; Nassenstein et al., 2010) and recipient neurons in the paratrigeminal nucleus connect extensively with thalamocortical networks involved in somatosensation, the results of this study have important broader implications for understanding the discriminative aspects of respiration and other visceral sensations.

Finally, the possibility that different circumstances govern glutamateric and peptidergic neurotransmission in the paratrigeminal nucleus may be important for understanding mechanisms for altered central nociceptive afferent processing in disease. For instance, given that peripheral inputs in healthy animals are predominately dependent upon non-peptidergic mechanisms (present study), how and when peptidergic neurotransmission occurs may be an important determinant for sensory plasticity. This could be especially relevant in conditions of laryngeal hypersensitivity in which altered neurochemistry of jugular-paratrigeminal afferent processing may be an underlying feature of sensory disturbances associated with respiratory disease. Accordingly, afferent sensitisation, phenotypic changes in sensory neurons and/or central alterations in afferent neurotransmission (Undem and Nassenstein, 2009; Undem et al., 2015; Mazzone and Undem, 2016; Driessen et al., 2017) will likely have 
a major impact on the nature of the output from the paratrigeminal nucleus and the ensuing reflex and behavioural respiratory responses that contribute to the symptoms of pulmonary disease. 


\section{Figure legends}

Figure 1. Identification and characterisation of guinea pig laryngeal jugular vagal ganglia sensory neurons and their terminations throughout the rostral-caudal extent of the paratrigeminal nucleus (Pa5). (A) A schematic depicting the experimental design of tracing from the larynx with $\mathrm{CT}_{-} \mathrm{B}_{594}$ (in red) and from the vagal ganglia with AAV-eGFP (in green). Example photomicrographs of a whole-mount preparation of the larynx showing the deposition of the retrograde neuronal tracer $\mathrm{CT}-\mathrm{B}_{594}$ in the dorsal laryngeal mucosa (top), and a vagal ganglia whole-mount preparation displaying the distribution of CT-B $\mathrm{B}_{594}$ retrogradely traced neurons predominately in the jugular (JG) rather than the nodose (NG) vagal ganglia (bottom). (B) Immunohistochemical characterisation of CT-B 594 traced jugular ganglia neurons showing populations of both C-fibre (CGRP and SP-expressing) and A-fibre (NF 160KD and vGlut1-expressing) laryngeal neurons. Quantitative cell counts (pie charts) show the proportion of the total traced neuron population positive for each immuno-marker assessed. Subsequently somal size analyses are presented as histograms (10 $\mathrm{mm}$ bins ) whereby the frequency is expressed relative to the total population of neurons that are both traced and immunopositive. Data were collated from $n=5$ jugular ganglia. (C) Representative photomicrographs of the rostro-caudal extent of the Pa5 showing brainstem hemisections immunostained for SP and vGlut1 on the left and the distribution of vagal afferent nerve terminals transduced by nodose vagal ganglia injections of AAV8 TRUFr-eGFP (AAVeGFP) in hemisections on the right. The higher magnification photomicrographs of the Pa5 (signified by red boxes) show the organisation and relative density of the corresponding afferent terminals in the Pa5. The top photomicrograph demonstrates AAV-eGFP transduced vagal afferents in close apposition to Pa5 neurons as stained by NeuN. Note the small number of NeuN positive Pa5 neurons and sparse afferent terminal labelling in the most rostral extent of this nucleus. The bottom photomicrograph displays the topographically distinct 
populations of SP and vGlut1 afferents in the Pa5. All scale bars represent $200 \mu \mathrm{m}$, unless stated otherwise. AP, area postrema; CGRP, Calcitonin Gene Related Peptide; CS, calamus scriptorious; IO, inferior olives; NF-160kD, Neurofilament 160kD; nTS, nucleus of the solitary tract; SP, substance P; Sp5, spinal trigeminal nucleus; vGlut1, vesicular glutamate transporter 1 .

Figure 2. Glutamatergic and peptidergic neurotransmission in the paratrigeminal nucleus (Pa5) mediate laryngeal-evoked respiratory reflexes. (A) Schematic representation of the vagal innervation of the guinea pig airways and the preparation used in the present physiological experiments. In the guinea pig the larynx is innervated by jugular ganglia (JG) derived afferents that travel primarily within the superior laryngeal nerves (SLN) and nodose ganglia (NG) derived afferents that travel primarily via the recurrent laryngeal nerves (RLN). Transection of the RLN's (represented with an X on the schematic) allows for jugularspecific reflexes to be evoked by electrical or chemical stimulation of the larynx. The representative physiological recording demonstrates the slowing of respiration with a small reduction in blood pressure and no change in heart rate, in response to electrical stimulation of the larynx at optimal stimulation parameters. (B) Electrical stimulation of the larynx, following vehicle (VEH) microinjection into the Pa5, evokes frequency-dependent changes in respiratory rate. A significant interaction effect $(p<0.0001)$ was observed between pharmacological modification in the Pa5 and frequency dependent respiratory slowing evoked by electrical stimulation of the larynx, indicating that bilateral microinjection of the ionotropic glutamate receptor antagonists CNQX/AP-5 $(\alpha \mathrm{GluR})$ or the neurokinin 1 and 3 receptor antagonists CP99994/ SB222200 ( $\alpha$ NKR) into the Pa5 is effective in altering laryngeal-evoked reflexes. Data represent the normalised mean \pm SEM for $n=30$ experiments, see Table 3 for further analyses. (C) Capsaicin superfusion across the laryngeal mucosal 
surface, after bilateral microinjection of $\mathrm{VEH}$ into the Pa5, evoked a reduction in respiratory rate. This respiratory slowing was significantly inhibited with bilateral microinjection of $+\alpha$ GluR in the rostro-caudal extent of the Pa5 $(*, p=0.02)$, but was unaffected following $+\alpha$ NKR bilateral microinjection. Data represent the mean \pm SEM change in respiration at the peak effect compared to immediately prior to stimulation, $n=10$ experiments. $A B P$, arterial blood pressure; AU, arbitrary units; $\mathrm{HR}$, heart rate and $\mathrm{TP}$, tracheal pressure; VN, vagus nerve.

Figure 3. Glutamate and capsaicin microinjection into the paratrigeminal nucleus (Pa5) resulted in differential respiratory responses. (A) Example trace of tracheal pressure (TP) during microinjection of 50mM DL-homocysteic acid (DLH, glutamate analogue, red arrow) into the caudal Pa5, evoking rapid but transient respiratory slowing. (B) Quantification of the peak change in respiratory rate following microinjection of vehicle (VEH) or DLH into either the caudal, central or rostral Pa5. *, $p=0.049$, significantly different to VEH in the caudal Pa5. (C) Representative trace of TP during microinjection of 400nM capsaicin (CAP, TRPV1 agonist, red arrow) into the Pa5, evoking a delayed, but persistent tachypnoea. (D) Quantification of the peak change in respiratory rate following microinjection of VEH or CAP into either the caudal, central or rostral Pa5. **, $p=0.009$, significantly different to VEH in the central Pa5. (E) Quantification of the effects of ionotropic glutamate receptor antagonists CNQX/AP-5 ( $\alpha$ GluR) or neurokinin 1 and 3 receptor antagonists CP99994/SB222200 ( $\alpha$ NKR) on peak changes in respiratory rate evoked by CAP microinjection into the central $\mathrm{Pa} 5 . * *, p=0.001$ significant attenuation of the response compared to CAP alone. Au, arbitrary units; TRPV1, transient receptor potential vanilloid 1. 
Figure 4. The guinea pig paratrigeminal nucleus (Pa5) connectome. (A) Schematic representation of the anterograde tracing experimental design. 10\% Biotin Dextran Amine (BDA) injections were made unilaterally into the Pa5 and traced terminals were assessed in $100 \mu \mathrm{m}$ intervals of the brainstem. The brainstem photomicrograph shows an example of BDA injection into the central Pa5 of the guinea pig. The central Pa5 injection site is also presented at a higher magnification to highlight BDA filled paratrigeminal neurons within the injection site. (B) A density weighted connectome diagram graphically presenting the quantification of ipsilaterally traced BDA terminals from the caudal, central and rostral subregions of the Pa5 to medullary and pontine nuclei. The weight of the interconnecting line between two regions corresponds to the extent of BDA labelling (sparse $=$ dotted line, intermediate $=$ solid line, dense $=$ thick solid line $).$ No labelled terminals were observed above the level of the pons. (C) Left hand side hemisection: choline acetyltransferase (ChAT) immunostaining of the ambiguus nucleus (Amb) as an anatomical landmark for distinguishing the ventrolateral medulla (VLM). Right hand side hemisection: representative photomicrograph of BDA traced terminals in the VLM. A high magnification photomicrograph of the area of the VLM confirming traced terminals in and around this region. (D) Left hand side hemisection: Forkhead box protein P2 (FoxP2) staining of the Kölliker-Fuse (KF) and parabrachial nuclei. Right hand side hemisection: representative photomicrograph of BDA traced terminals in the pontine nuclei. A high magnification representative photomicrograph of the KF, lateral parabrachial (LPB) nucleus and medial parabrachial (MPB) nucleus confirming dense terminal BDA labelling in these regions. The data represent the outcomes of $n=9$ successful BDA anterograde tracing experiments. Scale bars represent $1 \mathrm{~mm}$ in all images. $\mathrm{AP}$, area postrema; $\mathrm{Cu}$, Cuneate nuclei; $\mathrm{CS}$, calamus scriptorious; I5, intertrigeminal nucleus; IO, inferior olives; ml, medial lemniscus; Mo5, trigeminal motor nucleus; nTS, nucleus of the solitary tract; PR5, principal sensory trigeminal 
nucleus; PCRt, parvicellular reticular nucleus; Rt; reticular nuclei; scp; spinal cerebral peduncle; Sp5, spinal trigeminal nuclei; VII, facial nucleus.

Figure 5. The paratrigeminal nucleus (Pa5) consists of a heterogeneous population of neurons. (A) Example photomicrograph of a coronal section through the central paratrigeminal nucleus showing $\mathrm{CT}-\mathrm{B}_{488}$ and $\mathrm{CT}-\mathrm{B}_{594}$ retrograde labelled paratrigeminal neurons simultaneously traced from the ventral lateral medulla (VLM, arrows) and KöllikerFuse (KF, arrow heads) respectively. The higher magnification insert demonstrates clearly traced Pa5 neurons. Brainstem schematics depict the dual tracing experimental designs from the KF and VLM on the left and KF and lateral parabrachial nucleus (LPB) on the right. These correspond to quantified neuron counts of CT-B 488 and CT-B 594 single (green and red pie wedges) and dual (yellow pie wedge) labelled neurons in the Pa5. Only 8\% (81 out of 1007 neurons, $\mathrm{n}=4$ experiments) of Pa5 neurons were dual labelled from the KF and VLM and approximately 17\% (91 out of 529 neurons, $\mathrm{n}=3$ experiments) of Pa5 neurons were dual labelled from the KF and LPB. (B) Bar chart showing that calbindin (Calb) positive Pa5 neurons represent a significantly different population of neurons $(* * p=0.009)$ than the entire population represented by NeuN positive Pa5 neurons (data represents the mean \pm SEM; $n=3$ ). Photomicrograph of neurokinin 1 receptor (NK1R, arrows) and calbindin (Calb, arrow heads) immunoreactivity in the medulla $0.5 \mathrm{~mm}$ rostral to calamus scriptorius (CS), whereby the area corresponding to the Pa5 is highlighted by the red box. Quantified neuron counts were summed across $n=3$ experiments and are displayed using pie charts to show single labelled NK1R or Calb positive neurons (green and red wedges) and dual labelled neurons (yellow wedge). These data demonstrate that approximately 5.7\% (40 out of 702) of Pa5 neurons express both markers. In addition, representative photomicrographs are also presented to show evidence of both NK1R and Calb positive neurons at a high magnification within the 
Pa5. AP, area postrema; IO, inferior olives; nTS, nucleus of the solitary tract; Sp5, spinal trigeminal nucleus. 
Table 1: Stereotaxic co-ordinates for brainstem microinjection studies

\begin{tabular}{|c|c|c|c|}
\hline \multirow[b]{2}{*}{ Brainstem nucleus } & \multicolumn{3}{|c|}{ Stereotaxic co-ordinates $(\mathrm{mm})^{1}$} \\
\hline & $\begin{array}{l}\text { Anterior-Posterior } \\
\text { (AP) }\end{array}$ & $\begin{array}{l}\text { Medial-Lateral } \\
\text { (ML) }\end{array}$ & $\begin{array}{c}\text { Dorsal-Ventral } \\
\text { (DV) }\end{array}$ \\
\hline \multirow[t]{3}{*}{ Paratrigeminal $^{2}$} & Caudal $0.10 \pm 0.03$ & & \\
\hline & Central $0.50 \pm 0.03$ & $2.50 \pm 0.1$ & $1.30 \pm 0.2$ \\
\hline & Rostral $1.30 \pm 0.1$ & & \\
\hline Ventral Lateral Medulla ${ }^{2}$ & 1.53 & 1.95 & 4.60 \\
\hline Kölliker-Fuse ${ }^{3}$ & 12.35 & 2.62 & 9.15 \\
\hline Lateral Parabrachial $^{3}$ & 12.10 & 2.52 & 9.00 \\
\hline
\end{tabular}

${ }^{\mathrm{I}}$ The paratrigeminal nucleus was divided into three subregions encompassing the rostrocaudal extent of this nucleus to facilitate more precise anatomical and physiological investigations (de Sousa Buck et al., 2001; Pinto et al., 2006; Caous et al., 2014. ${ }^{2}$ Coordinates relative to calamus scriptorius. ${ }^{3}$ Co-ordinates relative to Bregma. 
Table 2: Primary antibody specifications

\begin{tabular}{llll} 
Antibody & Supplier & Host & Dilution \\
\hline Calbindin & Swant (CB-38a) & Rabbit & $1: 1000$ \\
Calcitonin Gene Related Peptide & Abcam (ab81887) & Mouse & $1: 1000$ \\
Choline Acetyltransferase & Chemicon (AB144P) & Goat & $1: 100$ \\
Forkhead Box Protein P2 & R\&D Systems (AF5647) & Sheep & $1: 10000$ \\
GFP Tag & Thermo Fisher (A11122) & Rabbit & $1: 1000$ \\
NeuN & Abcam (ab177487) & Rabbit & $1: 600$ \\
Neurofilament-160KD & Abcam (ab9034) & Rabbit & $1: 500$ \\
Neurokinin 1 Receptor & Chemicon (ab5060) & Rabbit & $1: 5000$ \\
Substance P & Millipore (AB5925) & Rat & $1: 200$ \\
Vesicular Glutamate Transporter 1 & Synaptic Systems (135302) & Rabbit & $1: 500$ \\
\hline
\end{tabular}


Table 3: The $\mathrm{E}_{\max }$ and $\mathrm{EF}_{50}$ following pharmacological modification of the paratrigeminal nucleus

\begin{tabular}{|c|c|c|}
\hline & $\begin{array}{c}\mathbf{E}_{\max } \text { (reduction in } \\
\text { breaths/min) }\end{array}$ & $\mathbf{E F}_{50}(\mathbf{H z})$ \\
\hline Vehicle & $42 \pm 3.8$ & $4.3 \pm 2.1$ \\
\hline CNQX/AP-5 & $17.3 \pm 3.1_{\# \mathrm{p}=0.01}^{* * * \mathrm{p}=0.0002}$ & $>32 \begin{array}{r}{ }^{*} \mathrm{p}=0.032 \\
\# \mathrm{p}=0.02\end{array}$ \\
\hline CP99994/SB222200 & $30.6 \pm 2.4^{* * \mathrm{p}=0.007}$ & $11 \pm 2.9$ \\
\hline BIBN4096 & $34.8 \pm 7.9$ & $2.2 \pm 0.5$ \\
\hline
\end{tabular}

* statistically compared to vehicle

\# statistically compared to CP99994/SB222200 


\section{References}

Alheid GF, Milsom WK, McCrimmon DR (2004) Pontine influences on breathing: an overview. Respir Physiol Neurobiol 143:104-114.

Alheid GF, Jiao W, McCrimmon DR (2011) Caudal nuclei of the rat nucleus of the solitary tract differentially innervate respiratory compartments within the ventrolateral medulla. Neuroscience 190:207-227.

Alioto OE, Lindsey CJ, Koepp J, Caous CA (2008) Sensory sciatic nerve afferent inputs to the dorsal lateral medulla in the rat. Auton Neurosci 140:80-87.

Altschuler SM, Bao XM, Bieger D, Hopkins DA, Miselis RR (1989) Viscerotopic representation of the upper alimentary tract in the rat: sensory ganglia and nuclei of the solitary and spinal trigeminal tracts. J Comp Neurol 283:248-268.

Bailey TW, Jin YH, Doyle MW, Andresen MC (2002) Vanilloid-sensitive afferents activate neurons with prominent A-type potassium currents in nucleus tractus solitarius. J Neurosci 22:8230-8237.

Bautista TG, Dutschmann M (2014) Inhibition of the pontine Kolliker-Fuse nucleus abolishes eupneic inspiratory hypoglossal motor discharge in rat. Neuroscience 267:22-29.

Bianchi AL, Gestreau C (2009) The brainstem respiratory network: an overview of a half century of research. Respir Physiol Neurobiol 168:4-12. 
Bongianni F, Mutolo D, Nardone F, Pantaleo T (2005) Ionotropic glutamate receptors mediate excitatory drive to caudal medullary expiratory neurons in the rabbit. Brain Res 1056: 145-157.

Canning BJ, Mazzone SB, Meeker SN, Mori N, Reynolds SM, Undem BJ (2004) Identification of the tracheal and laryngeal afferent neurones mediating cough in anaesthetized guinea-pigs. J Physiol 557:543-558.

Canning BJ, Mori N (2011) Encoding of the cough reflex in anesthetized guinea pigs. Am J Physiol Integr Comp Physiol 300:369-377.

Caous CA, Buck HdS, Lindsey CJ (2001) Neuronal connections of the paratrigeminal nucleus: a topographic analysis of neurons projecting to bulbar, pontine and thalamic nuclei related to cardiovascular, respiratory and sensory functions. Auton Neurosci 94:14-24.

Carstens E, Saxe I, Ralph R (1995) Brainstem neurons expressing c-Fos immunoreactivity following irritant chemical stimulation of the rat's tongue. Neuroscience 69:939-953.

Chan-Paley V (1978) The paratrigeminal nucleus. II. Identification and inter-relations of catecholamine axons, indoleamine axons and substance $\mathrm{P}$ immunoreactive cells in the neuropil. J Neurocytol 7:419-412. 
Chou Y-L, Scarupa MD, Mori N, Canning BJ (2008) Differential effects of airway afferent nerve subtypes on cough and respiration in anesthetized guinea pigs. Am J Physiol Regul Integr Comp Physiol 295:1572-1584.

Ciriello J, Hrycyshyn AW, Calaresu FR (1981) Glossopharyngeal and vagal afferent projections to the brain stem of the cat: a horseradish peroxidase study. J Auton Nerv Syst 4:63-79.

Corbett EK, Sinfield JK, McWilliam PN, Deuchars J, Batten TF (2005) Differential expression of vesicualr glutamate transporters by vagal afferent terminals in rat nucleus of the solitary tract: projections from the heart preferntially express vesicular glutamate transporter 1. Neuroscience 135: 133-145.

D'Autréaux F, Coppola E, Hirsch M-R, Birchmeier C, Brunet J-F (2011) Homeoprotein Phox $2 \mathrm{~b}$ commands a somatic-to-visceral switch in cranial sensory pathways. Pro Nati Acad Sci 108:20018-20023.

de Sousa Buck H, Caous CA, Lindsey CJ (2001) Projections of the paratrigeminal nucleus to the ambiguus, rostroventrolateral and lateral reticular nuclei, and the solitary tract. Auton Neurosci 87:187-200.

Dick TE, Bellingham MC, Richter DW (1994) Pontine respiratory neurons in anesthetized cats. Brain Res 636:259-269. 
Driessen AK, Farrell MJ, Mazzone SB, McGovern AE (2015) The Role of the Paratrigeminal Nucleus in Vagal Afferent Evoked Respiratory Reflexes: A Neuroanatomical and Functional Study in Guinea Pigs. Front Physiol 6:378.

Driessen AK, McGovern AE, Narula M, Yang SK, Keller JA, Farrell MJ, Mazzone SB (2017) Central mechanisms of airway sensation and cough hypersensitivity. Pulm Pharmacol Ther 47:9-15.

Dutschmann M, Dick TE (2012) Pontine mechanisms of respiratory control. Compr Physiol 2:2443-2469.

Dutschmann M, Herbert H (2006) The Kolliker-Fuse nucleus gates the postinspiratory phase of the respiratory cycle to control inspiratory off-switch and upper airway resistance in rat. Eur J Neurosci 24:1071-1084.

Fawley JA, Hofmann ME, Andresen MC (2016) Distinct Calcium Sources Support Multiple Modes of Synaptic Release from Cranial Sensory Afferents. J Neurosci 36:89578966.

Feil K, Herbert H (1995) Topographic organization of spinal and trigeminal somatosensory pathways to the rat parabrachial and Kolliker-Fuse nuclei. J Comp Neurol 353:506528.

Feldman JL, Mitchel GS, Nattie EE (2003) Breathing: rhythmicity, plasticity, chemosensitivity. Annu Rev Neurosci 26:239-266. 
Fenwick AJ, Wu S-w, Peters JH (2014) Isolation of TRPV1 independent mechanisms of spontaneous and asynchronous glutamate release at primary afferent to NTS synapses. Front Neurosci 8:6.

Geraghty DP, Mazzone SB (2002) Respiratory actions of vanilloid receptor agonists in the nucleus of the solitary tract: comparison of resiniferatoxin with non-pungent agents and anandamide. Br J Pharmacol 137:919-927.

Hermes SM, Colbert JF, Aicher SA (2015) Differential content of vesicular glutamate transporters in subsets of vagal afferents projecting to the nucleus tractus solitarii in the rat. J Comp Neurol 522:642-653

Hether S, Misono K, Lessard A (2013) The neurokinin-3 receptor $\left(\mathrm{NK}_{3} \mathrm{R}\right)$ antagonist SB222200 prevents the apomorphine-evoked surface but not nuclear $\mathrm{NK}_{3} \mathrm{R}$ redistribution in dopaminergic neurons of the rat ventral tegmental area. Neuroscience 247:12-24.

Hildreth CM, Goodchile AK (2010) Role of ionotropic GABA, glutamate and glycine receptors in the tonic and reflex control of cardiacl vagal outflow in the rat. Neuroscience 11:128-139.

Hofmann ME, Andresen MC (2016) Vanilloids selectively sensitize thermal glutamate release from TRPV1 expressing solitary tract afferents. Neuropharmacology 101:401411. 
Hunter DD, Undem BJ (1999) Identification and substance P content of vagal afferent neurons innervating the epithelium of the guinea pig trachea. Am J Respir Crit Care Med 159:1943-1948.

Ishikawa T, Isono S, Tanaka A, Tagaito Y, Nishino T (2005) Airway protective reflexes evoked by laryngeal instillation of distilled water under sevoflurane general anesthesia in children. Anesth Analg 101:1615-1618.

Junior AB, Caous CA, Yu Y-G, Lindsey CJ (2004) Barosensitive neurons in the rat tractus solitarius and paratrigeminal nucleus: a new model for medullary, cardiovascular reflex regulation. J Physiol Pharmacol 82:474-484.

Juranek I, Lembeck F (1997) Afferent C-fibres release substance P and glutamate. Can J Physiol Pharmacol 75:661-664.

Koepp J, Lindsey CJ, Motta EM, Rae GA (2006) Role of the paratrigeminal nucleus in nocifensive responses of rats to chemical, thermal and mechanical stimuli applied to the hind paw. Pain 122:235-244.

Kollarik M, Carr MJ, Ru F, Ring CJ, Hart VJ, Murdock P, Myers AC, Muroi Y, Undem BJ (2010) Transgene expression and effective gene silencing in vagal afferent neurons in vivo using recombinant adeno-associated virus vectors. J Physiol 588: 4303-4315. 
Kummer W, Fischer A, Kurkowski R, Heym C (1992) The sensory and sympathetic innervation of guinea-pig lung and trachea as studied by retrograde neuronal tracing and double-labelling immunohistochemistry. Neuroscience 49:715-737.

Kwong K, Kollarik M, Nassenstein C, Ru F, Undem BJ (2008) P2X2 receptors differentiate placodal vs. neural crest C-fiber phenotypes innervating guinea pig lungs and esophagus. Am J Physiol Lung Cell Mol Physiol 295:858-865.

Lieu T, Kollarik M, Myers AC, Undem BJ (2011) Neurotrophin and GDNF family ligand receptor expression in vagal sensory nerve subtypes innervating the adult guinea pig respiratory tract. Am J Physiol Lung Cell Mol Physiol 300:790-798.

Lindsey CJ, Buck HS, Fior-Chadi DR, Lapa RC (1997) Pressor effect mediated by bradykinin in the paratrigeminal nucleus of the rat. J Physiol 502:119-129.

Ma WL, Zhang WB, Feng G, Cai YL (2005) Calbindin D28k-containing neurons in the paratrigeminal nucleus receive convergent nociceptive information and project to nucleus of the solitary tract in rat. Brain Res 1038:132-140.

Ma WL, Zhang WB, Xiong KH, Guo F (2007) Visceral and orofacial somatic afferent fiber terminals converge onto the same neuron in paratrigeminal nucleus: An electron microscopic study in rats. Auton Neurosci 131:45-49. 
Mazzone SB, Geraghty DP (1999) Respiratory action of capsaicin microinjected into the nucleus of the solitary tract: involvement of vanilloid and tachykinin receptors. $\mathrm{Br} \mathrm{J}$ Pharmacol 127:473-481.

Mazzone SB, Canning BJ (2002) Evidence for differential reflex regulation of cholinergic and non-cholinergic parasyhmpathetic nerves innervating the airways. Am J Respir Crit Care Med 165: 1076-1083.

Mazzone S, McGovern A (2008) Immunohistochemical characterization of nodose cough receptor neurons projecting to the trachea of guinea pigs. Cough 4:9-25.

Mazzone SB, Undem BJ (2016) Vagal Afferent Innervation of the Airways in Health and Disease. Physiol Rev 96:975-1024.

McDougall SJ, Andresen MC (2013) Independent transmission of convergent visceral primary afferents in the solitary tract nucleus. J Neurophjysiol 109:507-517.

McGovern AE, Davis-Poynter N, Farrell MJ, Mazzone SB (2012) Transneuronal tracing of airways-related sensory circuitry using herpes simplex virus 1, strain H129. Neuroscience 207:148-166.

McGovern AE, Davis-Poynter N, Yang SK, Simmons DG, Farrell MJ, Mazzone SB (2015a) Evidence for multiple sensory circuits in the brain arising from the respiratory system: an anterograde viral tract tracing study in rodents. Brain Struct Funct 220:3683-3699. 
McGovern AE, Driessen AK, Simmons DG, Powell J, Davis-Poynter N, Farrell MJ, Mazzone SB (2015b) Distinct Brainstem and Forebrain Circuits Receiving Tracheal Sensory Neuron Inputs Revealed Using a Novel Conditional Anterograde Transsynaptic Viral Tracing System. J Neurosci 35:7041-7055.

Mutolo D, Bongianni F, Cinelli E, Fontana GA, Pantaleo T (2008) Modulation of the cough reflex by antitussive agents within the caudal aspect of the nucleus tractus solitarii in the rabbit. Am J Physiol Integr Comp Physiol 295:243-251

Nassenstein C, Taylor-Clark TE, Myers AC, Ru F, Nandigama R, Bettner W, Undem BJ (2010) Phenotypic distinctions between neural crest and placodal derived vagal Cfibres in mouse lungs. J Physiol 588:4769-4783.

Panneton WM, Burton H (1981) Corneal and periocular representation within the trigeminal sensory complex in the cat studied with tranganglionic transport of horseradish peroxidase. J Comp Neurol 199:327-344.

Panneton WM, Burton H (1985) Projections from the paratrigeminal nucleus and the medullary and spinal dorsal horns to the peribrachial area in the cat. Neuroscience 15:779-797.

Panneton VM, Pan B, Gan Q (2017) Somatotopy in the medullary dorsal horn as a basis for orofacial reflex behaviour. Front Neurol 8:1-13. 
Pedersen KE, Meeker SN, Riccio MM, Undem BJ (1998) Selective stimulation of jugular ganglion afferent neurons in guinea pig airways by hypertonic saline. J Appl Physiol $84: 499-506$

Phelan KD, Falls WM (1989) The interstitial system of the spinal trigeminal tract in the rat: anatomical evidence for morphological and functional heterogeneity. Somatosens Mot Res 6:367-399.

Pinto ML, de Cassia Machado R, Schoorlemmer GH, Colombari E, de Cassia Ribeiro da Silva Lapa R (2006) Topographic organization of the projections from the interstitial system of the spinal trigeminal tract to the parabrachial nucleus in the rat. Brain Res 1113:137-145.

Ramirez JM, Doi A, Garcia AJ $3^{\text {rd }}$, Elsen FP, Kock H, Wei AD (2012) The cellular building blocks of breathing. Compr Physiol 2:2683-2731.

Riccio MM, Kummer W, Biglari B, Myers AC, Undem BJ (1996) Interganglionic segregation of distinct vagal afferent fibre phenotypes in guinea-pig airways. J Physiol 496:521-530.

Rogoz K, Stjärne L, Kullander K, Lagerström MC (2015) VGLUT2 controls heat and punctuate hyperalgesia associated with nerve injury via TRPV1-Cre primary afferents. PLoS ONE 10:e0116568. 
Saxon DW, Hopkins DA (1998) Efferent and collateral organization of paratrigeminal nucleus projections: an anterograde and retrograde fluorescent tracer study in the rat. $\mathbf{J}$ Comp Neurol 402:93-110.

Smith JC, Abdala AP, Borgmann A, Rybak IA, Paton JF (2013) Brainstem respiratory networks: building blocks and microcircuits. Trends Neurosci 36:152-162.

Springall DR, Cadieux A, Oliveira H, Su H, Royston D, Polak JM (1987) Retrograde tracing shows that CGRP-immunoreactive nerves of rat trachea and lung originate from vagal and dorsal root ganglia. J Aunton Nerv Syst 20:155-166.

Stanic D, Dhingra RR, Dutschmann M (2018) Expression of the transcirption factor FoxP2 in brainstem respiratory circuits of adult rat is restricted to upper-airway pre-motor areas. Resp Physiol Neurobiol 250:14-18.

Tsujimura T, Udemgba C, Inoue M, Canning BJ (2013) Laryngeal and tracheal afferent nerve stimulation evokes swallowing in anaesthetized guinea pigs. J Physiol 591:46674679.

Undem BJ, Chuaychoo B, Lee M-G, Weinreich D, Myers AC, Kollarik M (2004) Subtypes of vagal afferent C-fibres in guinea-pig lungs. J Physiol 556:905-917.

Undem BJ, Nassenstein C (2009) Airway nerves and dyspnea associated with inflammatory airway disease. Resp Physiol Neurobiol 167:36-44. 
Undem BJ, Zaccone E, McGarvey L, Mazzone SB (2015) Neural dysfunction following respiratory viral infection as a cause of chronic cough hypersensitivity. Pulm Pharmacol Ther 33:52-56.

Yu YG, Caous CA, Balan AC, Rae GA, Lindsey CJ (2002) Cardiovascular responses to sciatic nerve stimulation are blocked by paratrigeminal nucleus lesion. Auton Neurosci 98:70-74.

Zhou Q, Imbe H, Dubner R, Ren K (1999) Persistent Fos protein expression after orofacial deep or cutaneous tissue inflammation in rats: implications for persistent orofacial pain. J Comp Neurol 412:276-291. 
A
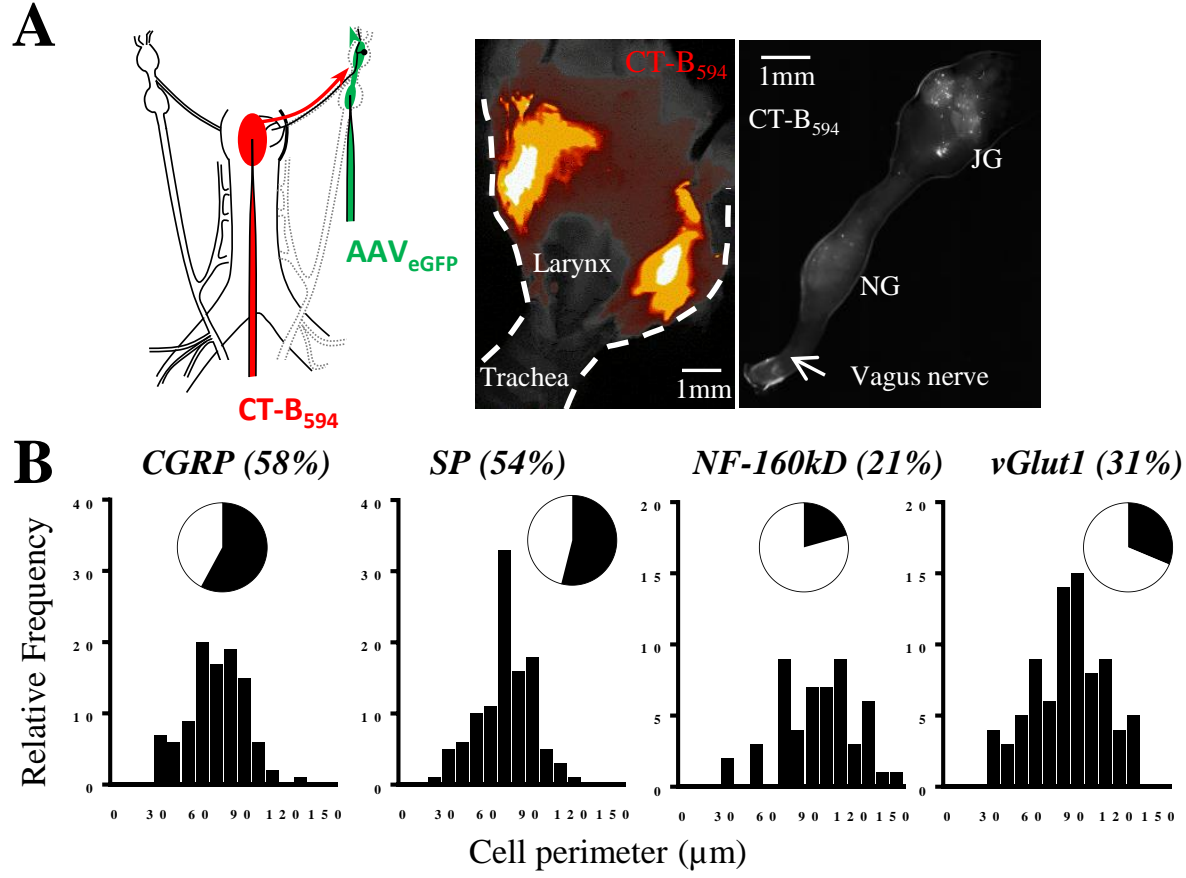

C

CAUDAL $(\mathrm{CS}+0.1 \mathrm{~mm})$

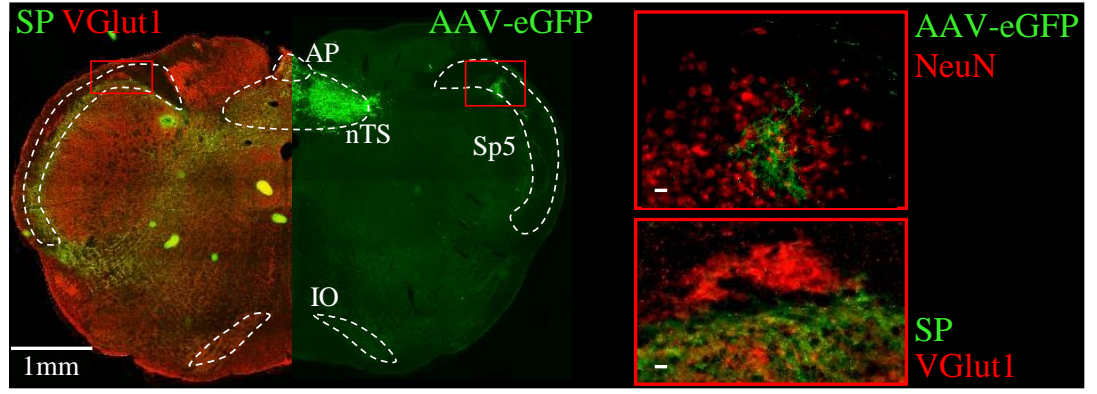

CENTRAL $(C S+0.5 \mathrm{~mm})$

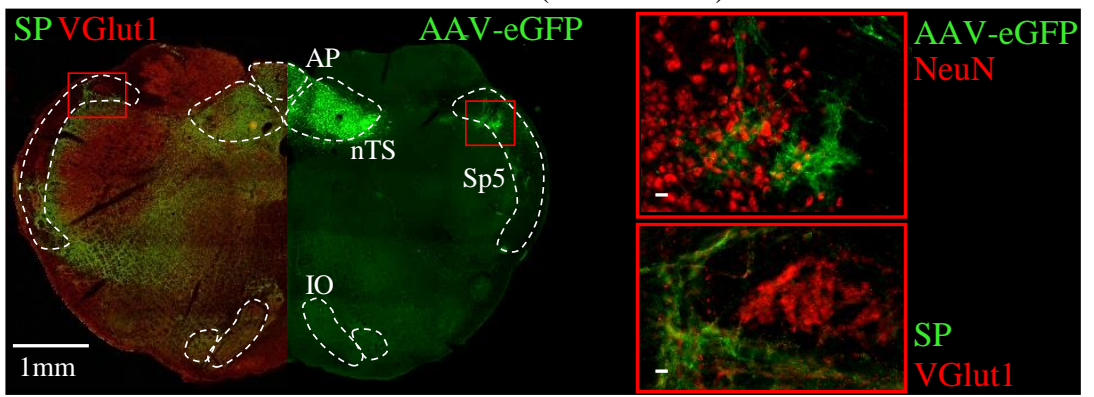

ROSTRAL $(C S+1.3 \mathrm{~mm})$

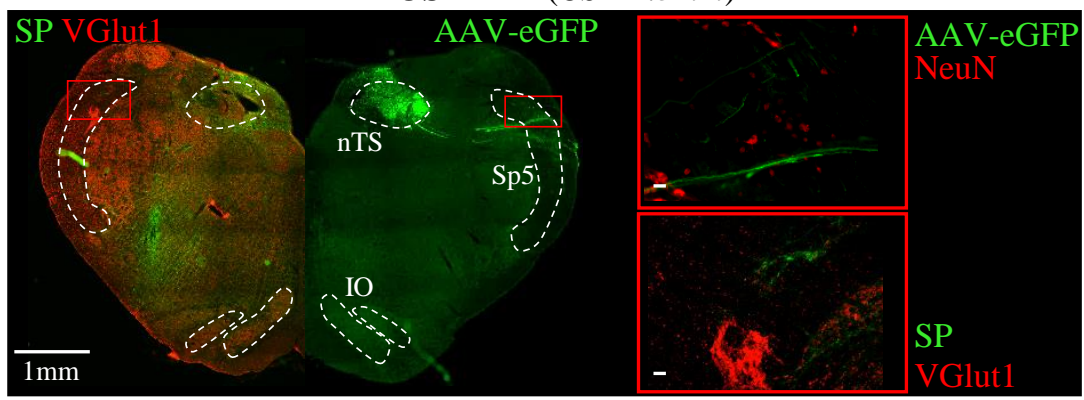


A

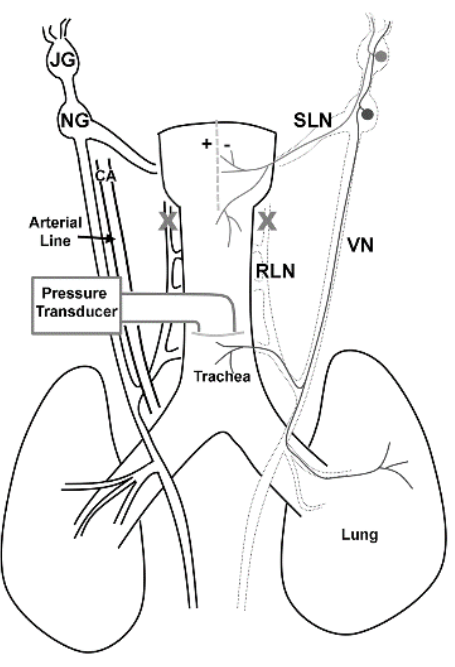

B

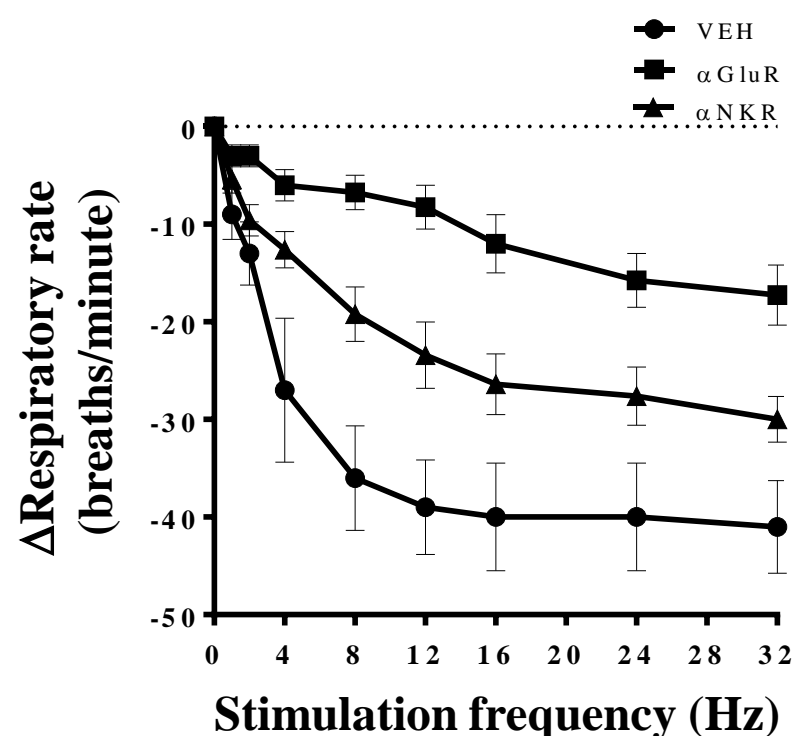

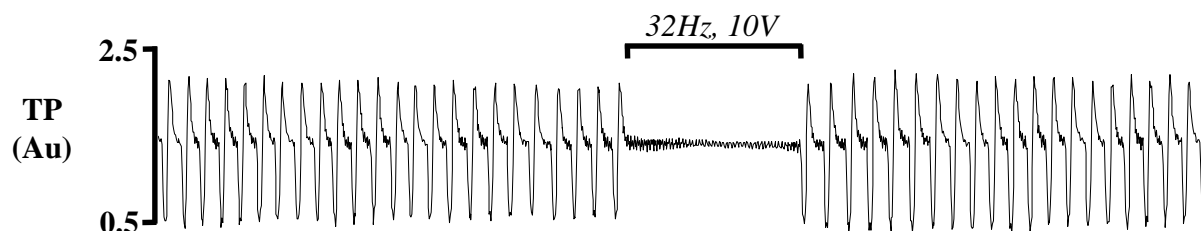

HR ${ }^{300}$ (BPM) 250

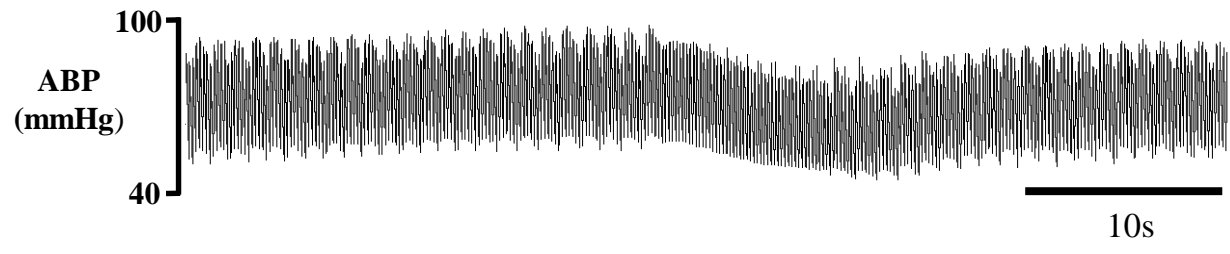

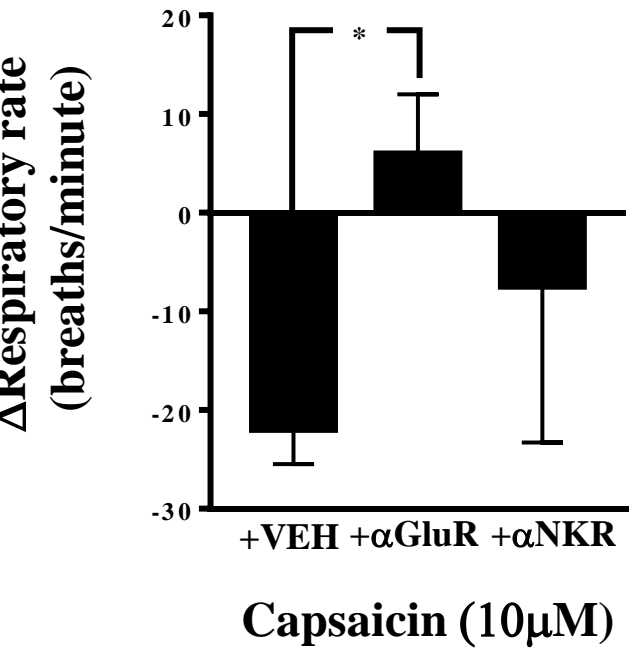


A DLH Microinjection

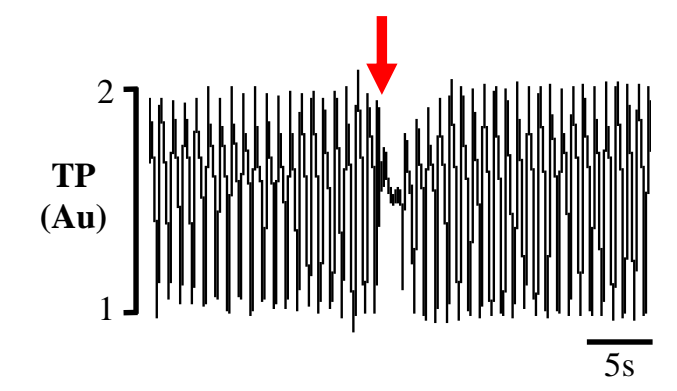

C CAP Microinjection
B Caudal
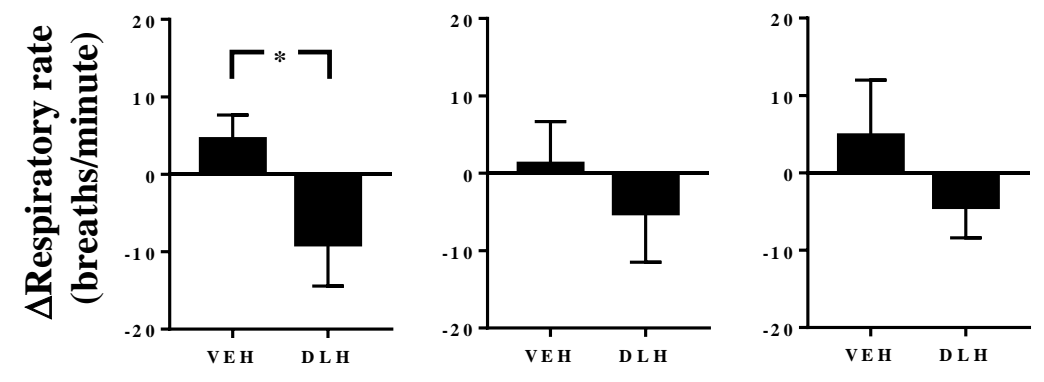

$\downarrow$

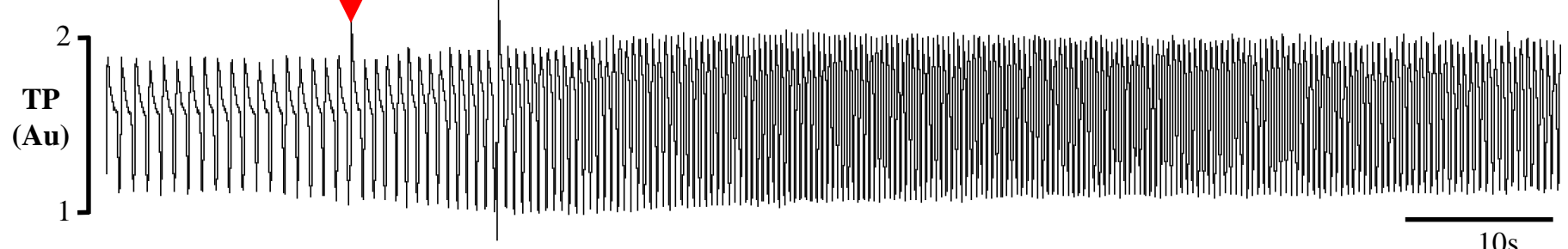

D CAUdal

ROSTRAL
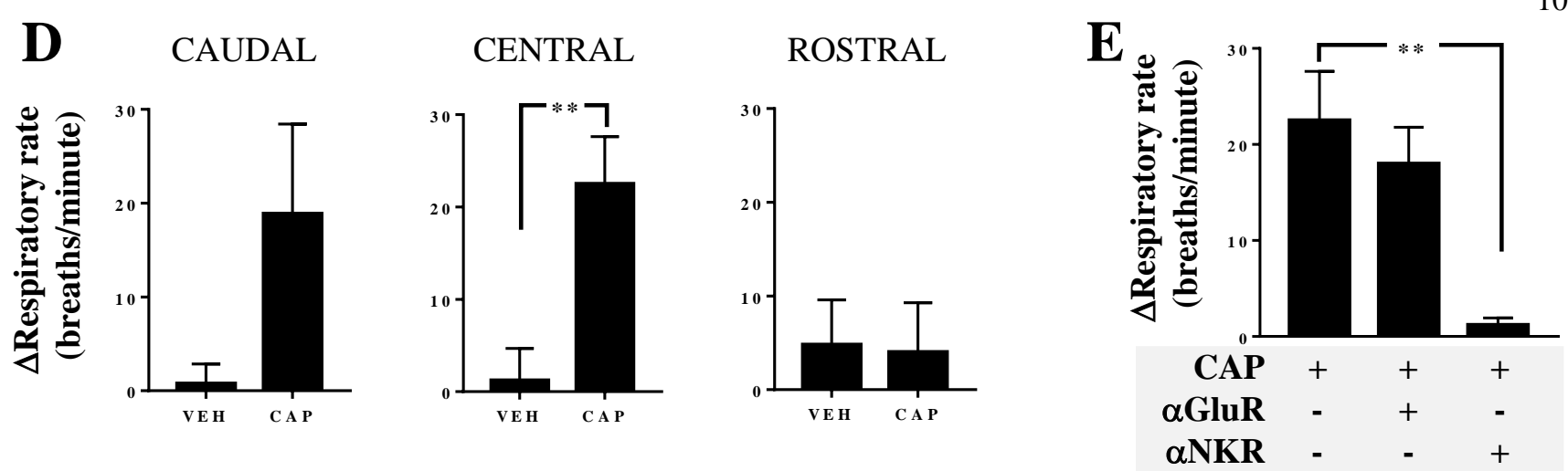
A

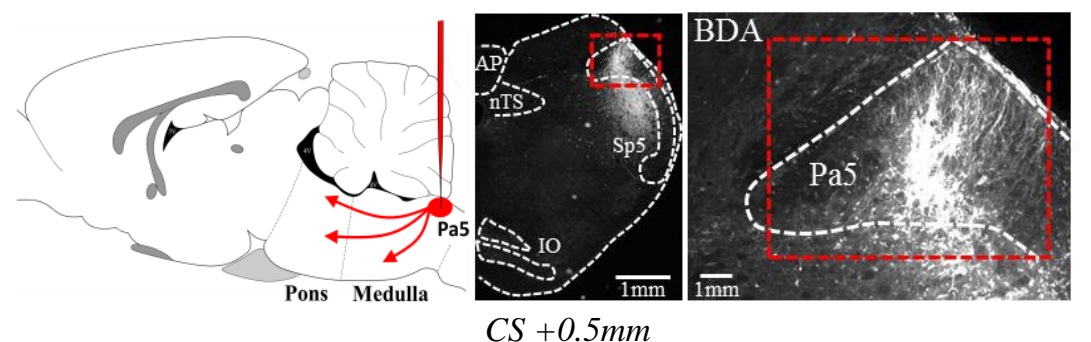

B

Medullary connectivity
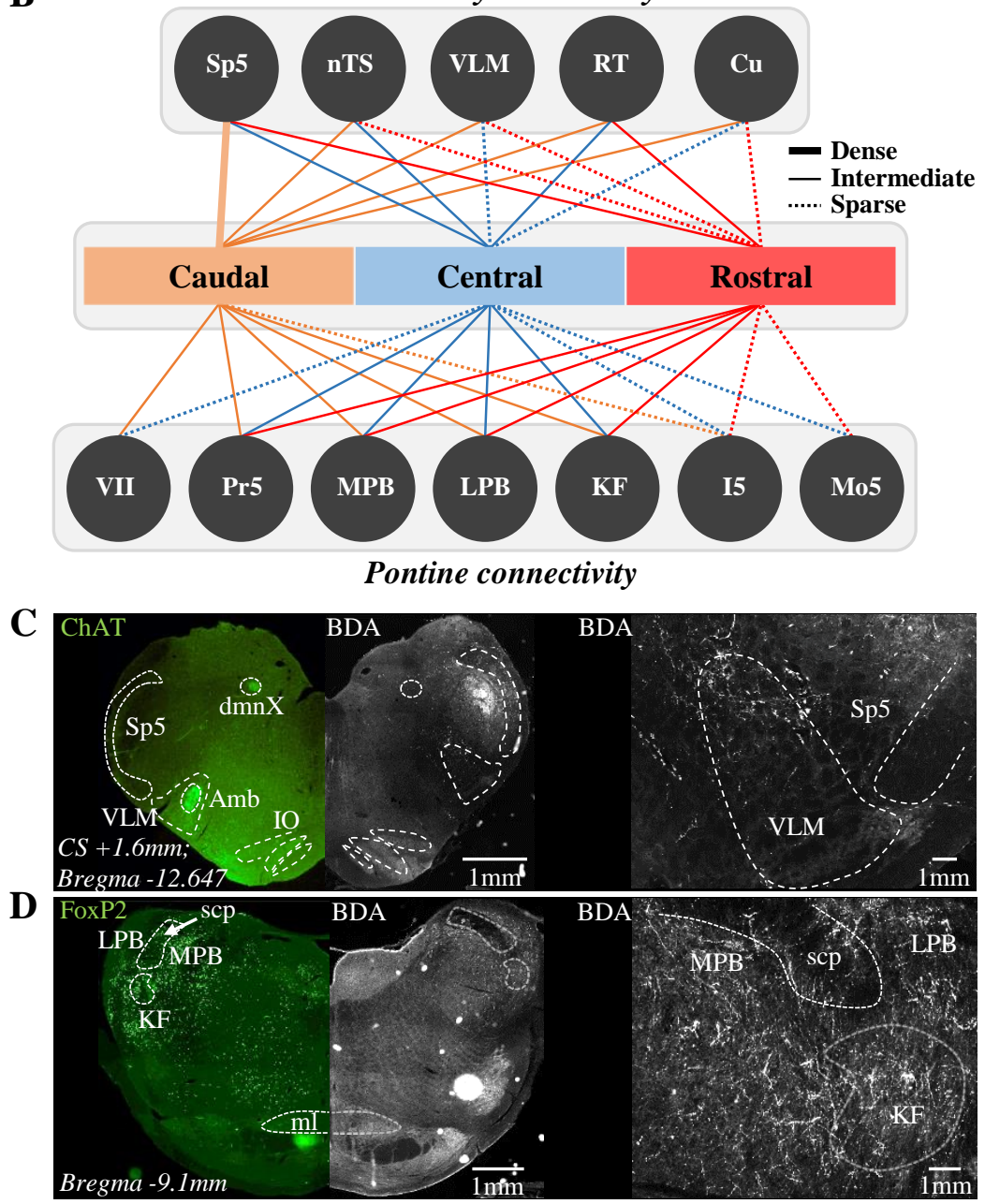

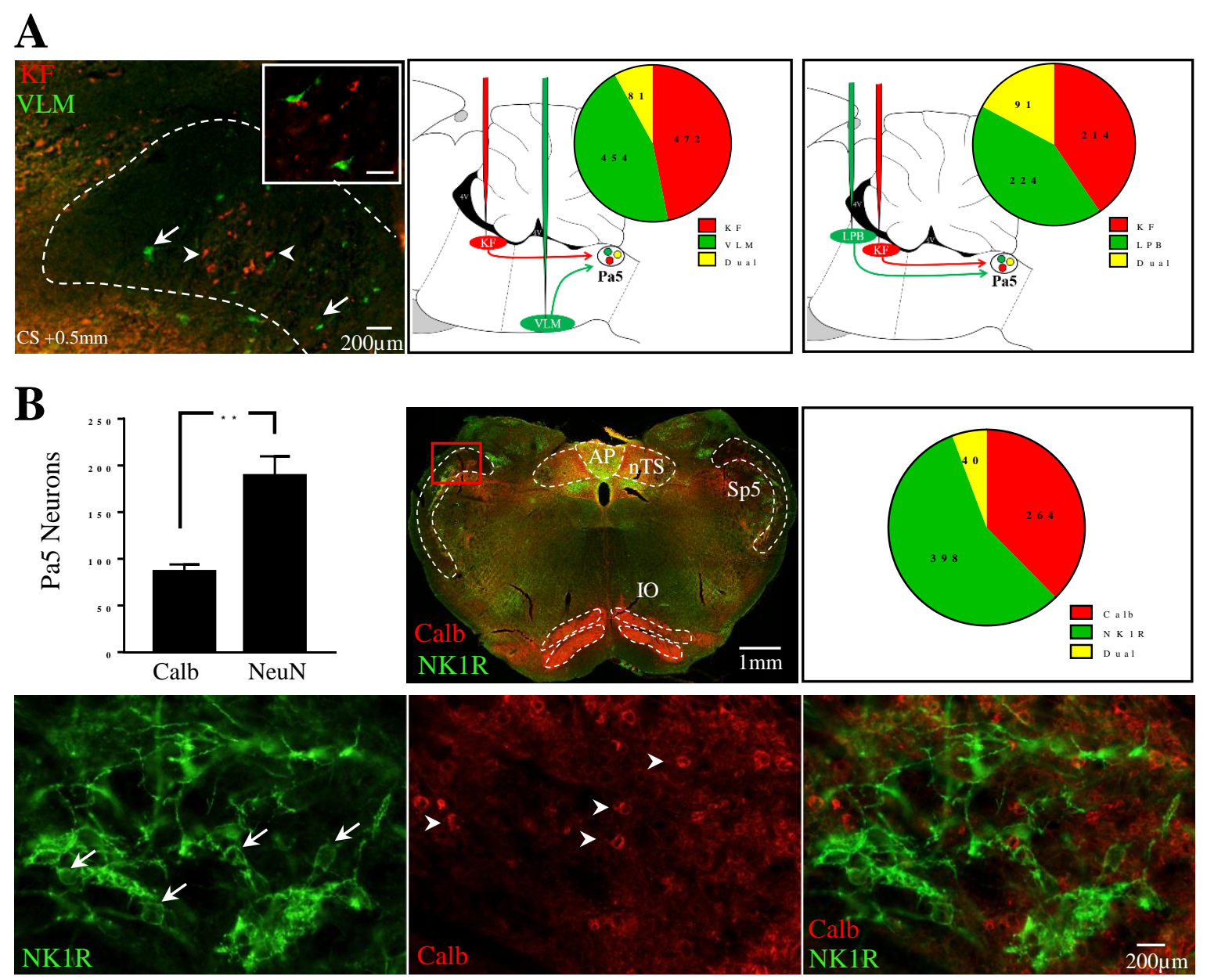


\section{University Library}

\section{- M M N E R VA A gateway to Melbourne's research publications}

Minerva Access is the Institutional Repository of The University of Melbourne

\section{Author/s:}

Driessen, AK;Farrell, MJ;Dutschmann, M;Stanic, D;McGovern, AE;Mazzone, SB

Title:

Reflex regulation of breathing by the paratrigeminal nucleus via multiple bulbar circuits

\section{Date:}

2018-12-01

Citation:

Driessen, A. K., Farrell, M. J., Dutschmann, M., Stanic, D., McGovern, A. E. \& Mazzone, S. B. (2018). Reflex regulation of breathing by the paratrigeminal nucleus via multiple bulbar circuits. BRAIN STRUCTURE \& FUNCTION, 223 (9), pp.4005-4022. https://doi.org/10.1007/ s00429-018-1732-Z.

Persistent Link:

http://hdl.handle.net/11343/282764 